\title{
A Utility-Based Welfare Criterion in a Model with Endogenous Capital Accumulation
}

\author{
Rochelle M. Edge \\ Division of Research and Statistics \\ Federal Reserve Board*
}

First Draft: August 5, 2003

This Draft: December 11, 2003

\begin{abstract}
This paper extends the utility-based welfare criterion developed by Rotemberg and Woodford (1997) and Woodford (2003) to a model with endogenous capital accumulation. The welfare criterion obtained for this model shares several features with the corresponding expressions that have been derived in simpler models without capital accumulation. In particular, a criterion can be specified such that welfare losses depend solely on quadratic functions of the model's variables, thus confirming that policy should be oriented toward stabilization of macroeconomic aggregates, rather than toward attaining particular levels of those aggregates. That said, an important difference that obtains in this case is that the composition of output directly affects welfare in the endogenous-capital model - a result that is not present in standard treatments.
\end{abstract}

${ }^{*}$ Rochelle M. Edge, Mail Stop 61, Federal Reserve Board, 20th and C Streets NW, Washington DC 20551. Email: rochelle.m.edge@frb.gov. I thank Thomas Laubach for many useful conversations and acknowledge with considerable gratitude the help provided by Jeremy Rudd, whose comments and substantial edits greatly improved upon previous drafts of this paper. The views expressed in this paper are my own and do not necessarily reflect the views of the Board of Governors or the staff of the Federal Reserve System. 


\section{Introduction}

Over the past two decades, considerable progress has been made in developing more rigorous methods with which to analyze monetary policy. The first important advance came twenty years ago, with the development of microfounded macromodels whose coefficients were directly related to deep structural parameters. These models had substantial potential application to policy analysis, since their microfounded structure rendered them immune to the Lucas critique, which had limited the usefulness of the previous generation of (reducedform) macromodels for policy evaluation. Later, once nominal rigidities were added to microfounded models, the way was opened for the analysis of monetary policy in a structural setup. The development of microfounded macromodels represents a fruitful avenue of research today, with particular focus being directed to the development of models that enjoy a greater degree of empirical validation. The structure of these models must necessarily be quite rich, with an explicit role for multiple spending aggregates (with differing sensitivities to real interest rate movements) and many sources of real rigidities, all of which yield better empirical fit.

Despite the development of increasingly sophisticated microfounded models, the welfare criteria against which alternative monetary policies were evaluated remained largely ad hoc for some time. Loss functions were often assumed to depend on the variance of some key macroeconomic variables - most often output and inflation - with no obvious link to household utility (the improvement of which is arguably the ultimate objective of stabilization policy). A major breakthrough came in the mid-1990s with the derivation of utility-based welfare criteria. ${ }^{1}$ Since loss functions constructed using this methodology were derived directly from the representative household's utility function, this allowed policy to be evaluated with the same degree of rigor that was being used to model the economy. Researchers were then able to consider how various features being incorporated into structural models to improve their empirical validity would influence the criteria against which alternative policies were evaluated.

One important limitation of this methodology remained, however: The procedure developed for constructing a utility-based welfare criterion in simple sticky-price models - and later implemented in more complicated models - remained confined to models in which there was no role for endogenous capital accumulation. As a result, ad hoc loss functions continued to be used to evaluate models with explicit investment and saving decisions. This drawback

\footnotetext{
${ }^{1}$ See Rotemberg and Woodford (1997).
} 
has placed an increasingly serious limitation on policy analysis in recent years, as models with endogenous capital accumulation — which enjoy much greater empirical validation - have become increasingly widespread.

This paper, by deriving a utility-based welfare criterion in a model with endogenous capital accumulation, demonstrates that the microfoundations of the welfare criteria used to evaluate policy in a model with endogenous capital can be made as rigorous as the specification of these models. In addition, I demonstrate the extent to which it is possible to preserve two features of the utility-based criteria that arise in simpler models: first, the (intuitively appealing) result that the welfare criterion can be expressed in terms of suitably defined "gap" terms; and second, the notion that welfare will depend only on quadratic functions of relevant variables, which implies in turn that variables' levels do not independently influence the welfare criterion and thus that policy should be directed at stabilization (as opposed to attaining particular target levels of economic variables).

As I demonstrate, the introduction of endogenous capital introduces a state variable to the model that significantly complicates the derivation of a utility-based welfare criterion relative to a model with fixed capital. In order to clearly describe what is required to derive a welfare criterion in an endogenous-capital model, the model I work with incorporates the simplest possible capital-evolution equation. In particular, I assume no adjustment costs (either in investment or capital) and no time-to-build, both of which have been used in recent years in order to improve the empirical fit of models with endogenous capital accumulation. (That said, I do point out where the introduction of these features would affect the derivation.)

The balance of the paper is organized as follows. Section 2 outlines the endogenous-capital model that I employ, while section 3 discusses different ways to define the "natural rate" of output and the capital stock in the context of this type of model. Next, section 4 constructs a second-order approximation to the model's utility function, which is then simplified in

section 5. Finally, section 6 discusses how fluctuations in price inflation enter the welfare criterion, and section 7 concludes.

\section{The Model}

Given that I am building upon an existing literature, the model that I consider shares most of the features of the canonical monetary business cycle model. The main purpose of this section, therefore, is to fix the details - and give the notation - for the particular specification 
I assume. An additional goal is to characterize the steady state and equilibrium conditions of the flexible-price version of the model, which will provide the natural-rate concepts that will be needed in order to compute the gap terms that enter the welfare criterion.

\subsection{Technologies and Preferences}

I begin by characterizing the production and utility relations that underpin firm and household behavior in my model economy.

\subsubsection{The Production Technology}

A single final good (denoted $Y_{t}$ ) is assumed to be produced through the aggregation of an infinite number of differentiated intermediate goods according to a Dixit-Stiglitz technology. Specifically, final goods production is given by the function

$$
Y_{t}=\left(\int_{0}^{1} Y_{t}(z)^{\frac{\theta-1}{\theta}} d z\right)^{\frac{\theta}{\theta-1}},
$$

where the variable $Y_{t}(z)$ denotes the quantity of the intermediate good $z$ (where $z \in[0,1]$ ) that is used to produce final output, and $\theta$ is the elasticity of substitution between the differentiated intermediate goods inputs used in the production of the final good.

The differentiated intermediate goods that are used as inputs in equation (1) are produced by combining labor $L_{t}$ and capital $K_{t}$ (which are both hired from households) according to a Cobb-Douglas production function. The production of intermediate good $z$ is represented by the function

$$
Y_{t}(z)=X_{t}\left(K_{t}(z)\right)^{\alpha}\left(H_{t}(z)\right)^{1-\alpha}
$$

where $X_{t}$ denotes a stationary unit-mean technology shock.

\subsubsection{The Evolution of the Capital Stock}

The economy's capital stock at the start of period $t+1, K_{t+1}$, is equal to the previous period's undepreciated capital stock, $(1-\delta) K_{t}$, augmented by new capital installed in the previous period, $I_{t}$. To keep the derivations as simple as possible, the assumed capital stock evolution

process does not include any adjustment costs. The evolution of the economy's capital stock is therefore given by

$$
K_{t+1}=(1-\delta) K_{t}+I_{t}
$$




\subsubsection{Preferences}

Households derive utility from their purchases of the consumption good $C_{t}$ and from the use of their leisure time (which is inversely related to the labor $H_{t}$ that they supply) according to the following utility function:

$$
E_{0}\left[\sum_{t=0}^{\infty} \beta^{t}\left(\frac{1}{1-\sigma}\left(C_{t}\right)^{1-\sigma} e^{a \chi_{t}^{c}}-\frac{1}{1+s}\left(H_{t}\right)^{1+s} e^{b \chi_{t}^{h}}\right)\right] .
$$

The parameter $\beta$ denotes the household's discount factor, $\sigma$ is the inverse of its intertemporal elasticity of substitution, and $s$ denotes its labor-supply elasticity. The stationary unitmean stochastic variables $\chi_{t}^{c}$ and $\chi_{t}^{h}$ represent aggregate shocks that affect the utility of consumption and disutility of labor, respectively.

\subsection{The Decentralized Flexible-Price Economy}

The model economy is comprised of the following set of agents. There is one representative, perfectly competitive firm in the final-good producing sector that purchases intermediate inputs from the continuum of intermediate-goods producers. The intermediate-goods producers, in turn, hire capital and labor from the representative household; these firms are monopolists, and are therefore able to set the price at which they supply their output. Finally, the household purchases the final good-which is used both for consumption and for augmenting the economy's capital stock - from the final-good producing firm.

\subsubsection{The Final-Goods Producer}

The competitive firm in the final-goods sector, which owns the production technology described in equation (1), takes as given the prices set by each intermediate-good producer for their differentiated output, $\left\{P_{t}(z)\right\}_{z=0}^{1}$, and chooses intermediate inputs $\left\{Y_{t}(z)\right\}_{z=0}^{1}$ so as to minimize the cost of producing its final output $Y_{t}$. The final-good producing firm therefore solves the problem:

$$
\min _{\left\{Y_{t}(z)\right\}_{z=0}^{1}} \int_{0}^{1} P_{t}(z) Y_{t}(z) d z \quad \text { s.t. } \quad Y_{t} \leq\left(\int_{0}^{1} Y_{t}(z)^{\frac{\theta-1}{\theta}} d z\right)^{\frac{\theta}{\theta-1}} .
$$

This implies a demand function for each intermediate good that is given by

$$
Y_{t}(z)=Y_{t}\left(\frac{P_{t}(z)}{P_{t}}\right)^{-\theta}
$$

The variable $P_{t}$, which denotes the aggregate price level in the intermediate goods sector, is given by $P_{t}=\left(\int_{0}^{1}\left(P_{t}(z)\right)^{1-\theta} d z\right)^{\frac{1}{1-\theta}}$. 


\subsubsection{Intermediate-Goods Producers}

Each intermediate-good producing firm $z \in[0,1]$ owns the production technology described in equation (2). In the cost-minimization step of firm $z$ 's problem, the firm takes as given the aggregate wage $W_{t}$ and the rental rate on capital $R_{t}^{k}$, and chooses aggregate labor $L_{t}(z)$ and capital $K_{t}(z)$ to minimize the costs of attaining its desired level of output $Y_{t}(z)$. Specifically, firm $z$ solves

$$
\min _{\left\{K_{t}(z), H_{t}(z)\right\}} R_{t}^{k} K_{t}(z)+W_{t} H_{t}(z) \quad \text { s.t. } Y_{t}(z)=X_{t}\left(K_{t}(z)\right)^{\alpha}\left(H_{t}(z)\right)^{1-\alpha} .
$$

Since each firm produces its own differentiated variety of intermediate output $Y_{t}(z)$, it is able to set its price $P_{t}(z)$, which it does taking into account the demand schedule for its output that it faces from the final-good sector (equation 6). In the flexible-price version of the model, intermediate-good producing firms solve their price-setting problem in the absence of any impediments. Hence, in the profit-maximizing part of its problem, firm $z$ takes as given the marginal cost $M C_{t}(z)$ for producing $Y_{t}(z)$, the aggregate price level $P_{t}$, and final output $Y_{t}$, and then chooses its price $P_{t}(z)$ to maximize its profits subject to the demand curve it faces for its differentiated output (equation 6). The profit-maximization part of the firm's problem is therefore described by:

$$
\max _{\left\{P_{t}(z)\right\}} P_{t}(z) Y_{t}(z)-(1-\tau) M C_{t}(z) Y_{t}(z) \quad \text { s.t. } Y_{t}(z)=Y_{t}\left(\frac{P_{t}(z)}{P_{t}}\right)^{-\theta},
$$

where $\tau$ is a subsidy equal to $\theta^{-1}$, which ensures that the steady-state equilibrium is equal to the Pareto-optimal equilibrium. The intermediate-good producing firm's profit maximization problem is the only part of the model that changes with the introduction of sticky prices; as it is not necessary to present the model with sticky prices at this stage, the discussion of this version of the model is postponed until section 6 .

\subsubsection{The Household}

The household has preferences over consumption and leisure that are given by equation (4), and can transform investment into productive capital according to equation (3). The household's holding of bonds, $B_{t}$, evolves according to:

$$
B_{t}=R_{t-1}^{-1} B_{t-1}+W_{t} H_{t}+R_{t}^{k} K_{t}+\text { Profits }_{t}+T_{t}-P_{t} C_{t}-P_{t} I_{t},
$$


where $R_{t}$ denotes the gross nominal interest rate and $T_{t}$ represents lump-sum transfers made to the household by the government (or, when negative, taxes levied on the household). ${ }^{2}$

The household takes as given the expected path of the gross nominal interest rate, the final-good price level $P_{t}$, the wage rate $W_{t}$, the capital rental rate $R_{t}^{k}$, profits income, and the initial bond stock $B_{0}$, and chooses consumption $C_{t}$, investment $I_{t}$ (and, hence, next period's capital stock $K_{t+1}$ ), and labor supply $L_{t}$ to maximize its utility subject to its budget constraint and the capital evolution equation. The household's problem can therefore be written as:

$$
\max _{\left\{C_{t}, I_{t}, H_{t}, K_{t+1}\right\}_{t=0}^{\infty}} E_{0}\left[\sum_{t=0}^{\infty} \beta^{t}\left(\frac{1}{1-\sigma}\left(C_{t}\right)^{1-\sigma} e^{a \chi_{t}^{c}}-\frac{1}{1+s}\left(H_{t}\right)^{1+s} e^{b \chi_{t}^{h}}\right)\right] \text { s.t. eqn.s }(3) \text { and }(9) \text {. }
$$

\subsection{Equilibrium in the Flexible-Price Economy}

Equilibrium is an allocation $\left\{C_{t}, I_{t}, Y_{t}, H_{t},\left\{H_{t}(z)\right\}_{z=0}^{1}, K_{t},\left\{K_{t}(z)\right\}_{z=0}^{1}\right\}_{t=0}^{\infty}$ and a sequence of values $\left\{W_{t} / P_{t}, R_{t}^{k} / P_{t},\left\{M C_{t}(z) / P_{t}\right\}_{z=0}^{1}, E_{t}\left(R_{t} P_{t} / P_{t+1}\right)\right\}_{t=0}^{\infty}$ that satisfy the following conditions: (i) the final-good producing firm solves (5); (ii) all intermediate-good producing firms solve (7) and (8); (iii) the household solves (10); (iv) the goods market clears; and, (v) all factor markets clear. Agents take the initial value of $K_{0}$ and the sequence of shocks $\left\{X_{t}, \chi_{t}^{c}, \chi_{t}^{h}\right\}_{t=0}^{\infty}$ that hit the economy as given.

\subsection{The Equilibrium Conditions of the Flexible-Price Economy}

The first-order condition from the final-good producing firm's cost-minimization problem is given by equation (6). The first-order conditions from the the intermediate-good producing firm's cost-minimization problem are given by:

$$
\begin{aligned}
\widetilde{R}_{t}^{k} & =\widetilde{M C}_{t}(z) \alpha \frac{Y_{t}(z)}{K_{t}(z)}, \\
\widetilde{W}_{t} & =\widetilde{M C}_{t}(z)(1-\alpha) \frac{Y_{t}(z)}{H_{t}(z)}, \text { and } \\
Y_{t}(z) & =X_{t}\left(K_{t}(z)\right)^{\alpha}\left(H_{t}(z)\right)^{1-\alpha},
\end{aligned}
$$

\footnotetext{
${ }^{2}$ To keep the government's role in the model as simple as possible, we assume that in each period lump-sum transfers exactly fund the subsidy payments that are made to intermediate-goods producers. This implies that the government's budget constraint is given by $\int_{0}^{1} \tau M C_{t}(z) Y_{t}(z) d z=-T_{t}$.
} 
where $\widetilde{R}_{t}^{k}=R_{t}^{k} / P_{t}, \widetilde{W}_{t}=W_{t} / P_{t}$, and $\widetilde{M C}_{t}(z)=M C_{t}(z) / P_{t}$. The first-order condition from the firm's profit-maximization problem is given by:

$$
P_{t}(z)=\left(\frac{\theta}{\theta-1}\right)(1-\tau) M C_{t}(z)=M C_{t}(z)
$$

which implies that

$$
\widetilde{M C} t(z)=1 \text { and } P_{t}=P_{t}(z)
$$

The first-order conditions from the household's utility-maximization problem are given by:

$$
\begin{aligned}
\frac{e^{a \chi_{t}^{c}}}{C_{t}^{\sigma}} & =\beta E_{t}\left[\frac{R_{t}}{\Pi_{t+1}} \cdot \frac{e^{a \chi_{t+1}^{c}}}{C_{t+1}^{\sigma}}\right], \\
\widetilde{W}_{t} & =\frac{H_{t}^{s} C_{t}^{\sigma} e^{b \chi_{t}^{h}}}{e^{a \chi_{t}^{c}}} \\
\left(1-E_{t}\left[\frac{\Pi_{t+1}}{R_{t}}\right]\right) & =\beta E_{t}\left[\frac{e^{a \chi_{t+1}^{c}} / C_{t+1}^{\sigma}}{e^{a \chi_{t}^{c}} / C_{t}^{\sigma}}\left(\frac{R_{t+1}^{k}}{P_{t+1}}-\delta\right)\right],
\end{aligned}
$$

and equation (3), where the variable $\Pi_{t+1}=P_{t+1} / P_{t}$ denotes the period $t+1$ gross inflation rate. Market clearing in the goods market requires

$$
Y_{t}=C_{t}+I_{t}
$$

while in factor markets it requires

$$
H_{t}=\int_{0}^{1} H_{t}(z) d z \text { and } K_{t}=\int_{0}^{1} K_{t}(z) d z
$$

\subsection{The Steady-State Conditions of the Flexible-Price Economy}

What follows is the steady-state solution for the economy (note that these steady-state conditions are the same in both the sticky- and flexible-price economies). The steady-state real and nominal interest rates are given by:

$$
\frac{R_{*}}{\Pi_{*}}=\frac{1}{\beta} \quad \text { and } \quad R_{*}=\frac{\Pi_{*}}{\beta},
$$

where the steady-state inflation rate is determined by the central bank's inflation target.

The steady-state values of real marginal cost $\left(\widetilde{M C}_{t}\right)$, the real rental rate $\left(\widetilde{R}_{t}^{k}\right)$, and the real wage $\left(\widetilde{W}_{t}\right)$ are:

$$
\widetilde{M C}_{*}=1, \quad \widetilde{R}_{*}^{k}=\frac{1}{\beta}-(1-\delta), \quad \text { and } \quad \widetilde{W}_{*}=(1-\alpha)\left(\frac{\alpha}{\frac{1}{\beta}-(1-\delta)}\right)^{\frac{\alpha}{1-\alpha}}
$$


while the steady-state ratios $\frac{L_{*}}{Y_{*}}, \frac{K_{*}}{Y_{*}}, \frac{I_{*}}{Y_{*}}$, and $\frac{C_{*}^{k}}{Y_{*}}$ are:

$$
\frac{L_{*}}{Y_{*}}=\left(\frac{1-\alpha}{\alpha}\right)^{\alpha}\left(\frac{\widetilde{R}_{*}^{k}}{\widetilde{W}_{*}}\right)^{\alpha}, \frac{K_{*}}{Y_{*}}=\left(\frac{\alpha}{1-\alpha}\right)^{1-\alpha}\left(\frac{\widetilde{W}_{*}}{\widetilde{R}_{*}^{k}}\right)^{1-\alpha}, \frac{I_{*}}{Y_{*}}=\frac{\delta K_{*}}{Y_{*}} \text {, and } \frac{C_{*}}{Y_{*}}=1-\frac{\delta K_{*}}{Y_{*}} \text {. }
$$

From equation (17) we know that

$$
Y_{*}=\left(W_{*}\right)^{\frac{1}{s+\sigma}}\left(\frac{H_{*}}{Y_{*}}\right)^{\frac{-s}{s+\sigma}}\left(\frac{C_{*}}{Y_{*}}\right)^{\frac{-\sigma}{s+\sigma}}
$$

which allows us to calculate steady-state values of $L_{*}, K_{*}, I_{*}$, and $C_{*}$ from the ratios defined above.

In addition, note for future reference that the steady-state version of equation (17) can be written as:

$$
\frac{H_{*}^{s}}{C_{*}^{-\sigma}}=\widetilde{W}_{*} \quad \text { or } \quad \frac{H_{*}^{1+s}}{C_{*}^{1-\sigma}}=\widetilde{W}_{*} \frac{H_{*}}{C_{*}}=\widetilde{W}_{*} \frac{H_{*}}{Y_{*}} \cdot \frac{Y_{*}}{C_{*}} .
$$

We know from equation $(12)$ that $\widetilde{W}_{*}=(1-\alpha) \frac{Y_{*}}{H_{*}}$, so that $\widetilde{W}_{*} \frac{H_{*}}{Y_{*}}=(1-\alpha)$. This implies that

$$
\frac{H_{*}^{1+s}}{C_{*}^{1-\sigma}}=\frac{1-\alpha}{C_{*} / Y_{*}} \quad \text { or } \quad \frac{H_{*}^{1+s}}{1-\alpha}=\frac{C_{*}^{1-\sigma}}{C_{*} / Y_{*}} .
$$

An additional steady-state relationship that we will refer to later is

$$
\frac{I_{*}}{Y_{*}}=\frac{\delta K_{*}}{Y_{*}}=\frac{\delta \widetilde{R}_{*}^{k} K_{*}}{Y_{*}} \cdot \frac{1}{\widetilde{R}_{*}^{k}} .
$$

We know from equation (11) that $\widetilde{R}_{*}^{k}=\alpha \frac{Y_{*}}{K_{*}}$, so $\widetilde{R}_{*}^{k} \frac{K_{*}}{Y_{*}}=\alpha$; moreover, we know that the steady-state value of $\widetilde{R}_{*}^{k}$ is $\frac{1}{\beta}-(1-\delta)$. Together, these imply that

$$
\frac{I_{*}}{Y_{*}}=\frac{\delta \alpha \beta}{1-\beta(1-\delta)} .
$$

\subsection{Log-Linearized Equilibrium Conditions in the Flexible-Price Economy}

The first-order conditions from the intermediate-good producing firm's cost-minimization problem (equations 11, 12, and 13) log-linearize to:

$$
\begin{aligned}
r_{t}^{k} & =m c_{t}+y_{t}-k_{t}+\mathcal{O}\left(\|\xi\|^{2}\right), \\
w_{t} & =m c_{t}+y_{t}-h_{t}+\mathcal{O}\left(\|\xi\|^{2}\right), \quad \text { and } \\
y_{t} & =x_{t}+\alpha k_{t}+(1-\alpha) h_{t}+\mathcal{O}\left(\|\xi\|^{2}\right),
\end{aligned}
$$


where $r_{t}^{k}, w_{t}$, and $m c_{t}$ represent the log deviations of the real rental rate, the real wage, and real marginal cost from their respective steady-state levels (which are defined in section 2.5), and $x_{t}$ is the log of the technology shock. The first-order condition from the intermediategood producing firm's profit-maximization problem (equation 14) log-linearizes to

$$
m c_{t}=0+\mathcal{O}\left(\|\xi\|^{2}\right)
$$

(Note that equation 26 is the only equation of the model that will change when sticky prices are introduced.) The first-order conditions from the household's utility-maximization problem (equations 3, 16, 17, and 18) log-linearize to

$$
\begin{aligned}
a^{\prime} \chi_{t}^{c}-\sigma c_{t} & =\left(r_{t}-E_{t} \pi_{t+1}\right)+a^{\prime} E_{t} \chi_{t+1}^{c}-E_{t} \sigma c_{t+1}+\mathcal{O}\left(\|\xi\|^{2}\right), \\
w_{t} & =s h_{t}+\sigma c_{t}+b \chi_{t}^{h}-a \chi_{t}^{c}+\mathcal{O}\left(\|\xi\|^{2}\right), \\
E_{t} r_{t+1}^{k} & =\frac{1}{1-\beta(1-\delta)}\left(r_{t}-E_{t} \pi_{t+1}\right)+\mathcal{O}\left(\|\xi\|^{2}\right), \quad \text { and } \\
k_{t+1} & =(1-\delta) k_{t}+\delta i_{t}+\mathcal{O}\left(\|\xi\|^{2}\right) .
\end{aligned}
$$

Finally, the goods-market clearing condition log-linearizes to

$$
y_{t}=\mathrm{c} c_{t}+(1-\mathrm{c}) i_{t}+\mathcal{O}\left(\|\xi\|^{2}\right)
$$

where $\mathrm{c}$ denotes the steady-state share of consumption in aggregate output (i.e., c $\equiv C_{*} / Y_{*}$ ), an expression for which was derived in section 2.4 .

\section{Defining the Natural Rate of Output}

Our second-order approximation to the utility function will be derived in terms of output (which is the obvious variable of interest) and the capital stock (which will be our state variable). In addition, wherever possible we will want to express all variables in the approximate welfare criterion as "gap" terms - i.e., relative to the variable's natural rate (defined as the level of the variable that would prevail under fully flexible prices).

For our purposes, it is useful to consider the relationship between the shock variables in the model and output and the capital stock. There are two such relationships, which come from the model's first-order conditions; the first combines equations (24), (25), (28), and (31) to yield

$$
a \chi_{t}^{c}-b \chi_{t}^{h}+\frac{1+s}{1-\alpha} x_{t}=\left[\frac{s+\alpha}{1-\alpha}+\frac{\sigma}{\mathrm{c}}\right] y_{t}-\left[\frac{\alpha(1+s)}{1-\alpha}\right] k_{t}-\left[\frac{\sigma}{\mathrm{c}}(1-\mathrm{c})\right] i_{t}+\mathcal{O}\left(\|\xi\|^{2}\right),
$$


while the second combines equations (23), (27), (29), and (31) to yield

$a^{\prime} \chi_{t}^{c}=a^{\prime} E_{t} \chi_{t+1}^{c}+(1-\beta(1-\delta))\left(E_{t} y_{t+1}-E_{t} k_{t+1}\right)-\frac{\sigma}{\mathrm{c}}\left(E_{t} y_{t+1}-y_{t}\right)+\frac{\sigma}{\mathrm{c}}(1-\mathrm{c})\left(i_{t+1}-i_{t}\right)+\mathcal{O}\left(\|\xi\|^{2}\right)$.

The $i_{t}$ term that appears in in both equations (32) and (33) is related to current and next period's capital stock by $(30){ }^{3}$

Equations (32) and (33) apply only to the flexible-price version of the model; as such, the model variables in these equations are (by definition) largely natural rate concepts (which will be labeled with a superscript $n$ ). In defining the natural rate variables, however, we face the complication that there currently exist two competing definitions of the natural rate of output in models with endogenous capital accumulation. The difference hinges on whether the natural rate of output in a given period, which now depends on the capital stock in addition to the shocks that hit the economy, should be defined based on the capital stock consistent with the flexible-price model (that is, $k_{t}^{n}$ ), or the capital stock that actually exists in the economy in that period (that is, the capital stock from the sticky-price model, $k_{t}$ ).

The first concept has been advocated by Neiss and Nelson (2003), who construct their definition of the natural rate of output on the basis that the relevant capital stock is that which would have been in place had the economy always existed in a flexible-price world. To be precise, while the initial capital stock $k_{0}$ is given, the capital stock that defines the natural rate of output in all subsequent periods is that from the flexible-price model, which is denoted by $\left\{k_{t+1}^{n}\right\}_{t=0}^{\infty}$. Neiss and Nelson's definition therefore implies that the flexible-price levels of output and the capital stock are related to the economy's shocks according to the following sets of relations:

$$
\begin{gathered}
a \chi_{t}^{c}-b \chi_{t}^{h}+\frac{1+s}{1-\alpha} x_{t}=\left[\frac{s+\alpha}{1-\alpha}+\frac{\sigma}{\mathrm{c}}\right] y_{t}^{n}-\left[\frac{\alpha(1+s)}{1-\alpha}\right] k_{t}^{n}-\left[\frac{\sigma}{\mathrm{c}}(1-\mathrm{c})\right] i_{t}^{n}+\mathcal{O}\left(\|\xi\|^{2}\right), \quad \text { and } \\
a^{\prime} \chi_{t}^{c}=a^{\prime} E_{t} \chi_{t+1}^{c}+(1-\beta(1-\delta))\left(E_{t} y_{t+1}^{n}-E_{t} k_{t+1}^{n}\right)-\frac{\sigma}{\mathrm{c}}\left(E_{t} y_{t+1}^{n}-y_{t}^{n}\right)+\frac{\sigma}{\mathrm{c}}(1-\mathrm{c})\left(E_{t} i_{t+1}^{n}-i_{t}^{n}\right)+\mathcal{O}\left(\|\xi\|^{2}\right),
\end{gathered}
$$

where

$$
i_{t}^{n}=\left(\frac{1}{\delta}\right) k_{t+1}^{n}-\left(\frac{1-\delta}{\delta}\right) k_{t}^{n}+\mathcal{O}\left(\|\xi\|^{2}\right)
$$

\footnotetext{
${ }^{3}$ As noted earlier, we have kept the derivations in the endogenous-capital model as simple as possible by assuming a capital stock evolution process (given by equation 3) that lacks any form of adjustment costs; this delivers a very simple (log-linearized) capital supply relation (see equation 29). Richer and more realistic capital accumulation assumptions would imply more complicated versions of equation (29) with either additional capital-stock or investment-spending terms. Such terms would then appear in equation (33), and thus alter our definitions of the natural rate of output. For the most part, this is the stage at which alternative capital accumulation technologies would change our derivation of the welfare criterion.
} 
Hence, we have that

$a \chi_{t}^{c}-b \chi_{t}^{h}+\frac{1+s}{1-\alpha} x_{t}=\left[\frac{s+\alpha}{1-\alpha}+\frac{\sigma}{\mathrm{c}}\right] y_{t}^{n}-\left[\frac{\alpha(1+s)}{1-\alpha}-\frac{\sigma}{\mathrm{c}} \cdot \frac{1-\mathrm{c}}{\delta}(1-\delta)\right] k_{t}^{n}-\left[\frac{\sigma}{\mathrm{c}} \cdot \frac{1-\mathrm{c}}{\delta}\right] k_{t+1}^{n}+\mathcal{O}\left(\|\xi\|^{2}\right)$,

and

$$
\begin{aligned}
a^{\prime} \chi_{t}^{c}= & a^{\prime} E_{t} \chi_{t+1}^{c}+(1-\beta(1-\delta))\left(E_{t} y_{t+1}^{n}-E_{t} k_{t+1}^{n}\right)-\frac{\sigma}{c}\left(E_{t} y_{t+1}^{n}-y_{t}^{n}\right) \\
& +\frac{\sigma}{c} \cdot \frac{1-c}{\delta}\left(E_{t} k_{t+2}^{n}-k_{t+1}^{n}\right)-\frac{\sigma}{c} \cdot \frac{1-c}{\delta}(1-\delta)\left(k_{t+1}^{n}-k_{t}^{n}\right)+\mathcal{O}\left(\|\xi\|^{2}\right) .
\end{aligned}
$$

In contrast, Woodford's (2003) definition of the natural rate of output is constructed based on the actual capital stock with which the economy enters each period. Thus, if the model that represents how the economy operates in practice is the sticky-price model, then it is the period- $t$ capital stock from that model that determines the current natural rate of output. Woodford's definition therefore implies that the flexible-price levels of output and the capital stock will be related to the model's shock terms by:

$$
\begin{gathered}
a \chi_{t}^{c}-b \chi_{t}^{h}+\frac{1+s}{1-\alpha} x_{t}=\left[\frac{s+\alpha}{1-\alpha}+\frac{\sigma}{\mathrm{c}}\right] y_{t}^{n}-\left[\frac{\alpha(1+s)}{1-\alpha}\right] k_{t}-\left[\frac{\sigma}{\mathrm{c}}(1-\mathrm{c})\right] i_{t}^{n}+\mathcal{O}\left(\|\xi\|^{2}\right), \quad \text { and } \\
a^{\prime} \chi_{t}^{c}=a^{\prime} E_{t} \chi_{t+1}^{c}+(1-\beta(1-\delta))\left(E_{t} y_{t+1}^{n}-E_{t} k_{t+1}^{n}\right)-\frac{\sigma}{\mathrm{c}}\left(E_{t} y_{t+1}^{n}-y_{t}^{n}\right)+\frac{\sigma}{\mathrm{c}}(1-\mathrm{c})\left(E_{t} i_{t+1}^{n}-i_{t}^{n}\right)+\mathcal{O}\left(\|\xi\|^{2}\right),
\end{gathered}
$$

where

$$
i_{t}^{n}=\left(\frac{1}{\delta}\right) k_{t+1}^{n}-\left(\frac{1-\delta}{\delta}\right) k_{t} .
$$

As a result,

$a \chi_{t}^{c}-b \chi_{t}^{h}+\frac{1+s}{1-\alpha} x_{t}=\left[\frac{s+\alpha}{1-\alpha}+\frac{\sigma}{\mathrm{c}}\right] y_{t}^{n}-\left[\frac{\alpha(1+s)}{1-\alpha}-\frac{\sigma}{\mathrm{c}} \cdot \frac{1-\mathrm{c}}{\delta}(1-\delta)\right] k_{t}-\left[\frac{\sigma}{\mathrm{c}} \cdot \frac{1-\mathrm{c}}{\delta}\right] k_{t+1}^{n}+\mathcal{O}\left(\|\xi\|^{2}\right)$,

and

$$
\begin{aligned}
a^{\prime} \chi_{t}^{c}= & a^{\prime} E_{t} \chi_{t+1}^{c}+(1-\beta(1-\delta))\left(E_{t} y_{t+1}^{n}-E_{t} k_{t+1}^{n}\right)-\frac{\sigma}{\mathrm{c}}\left(E_{t} y_{t+1}^{n}-y_{t}^{n}\right) \\
& +\frac{\sigma}{\mathrm{c}} \cdot \frac{1-\mathrm{c}}{\delta}\left(E_{t} k_{t+2}^{n}-k_{t+1}^{n}\right)-\frac{\sigma}{\mathrm{c}} \cdot \frac{1-\mathrm{c}}{\delta}(1-\delta)\left(k_{t+1}-k_{t}\right)+\mathcal{O}\left(\|\xi\|^{2}\right) .
\end{aligned}
$$

The difference, of course, reflects the assumption that when we enter period $t+1$, it is the capital stock that is actually present that determines how $i_{t+1}^{n}$ is defined; hence, $i_{t+1}^{n}$ will equal $\left(\frac{1}{\delta}\right) k_{t+2}^{n}-\left(\frac{1-\delta}{\delta}\right) k_{t+1}$ under this definition of the natural rate.

As Woodford argues, Neiss and Nelson's definition of the natural rate of output renders irrelevant the capital stock that actually exists and its effect on the economy's productive 
capacity, and instead defines the natural rate of output conditional on some hypothetical capital stock. Woodford's definition of the natural rate of output - which is based on the actual capital stock - is therefore more akin to what is commonly thought of as "potential" output. Nevertheless, from a technical standpoint, the Neiss-Nelson definition carries an important advantage inasmuch as it is completely symmetric, which - as we will see - makes the derivation of the loss function considerably cleaner. We therefore consider both natural rate definitions in what follows.

We have already noted that in the model with endogenous capital accumulation, the capital stock enters our expression for the natural rate of output (equations 34 or 36) along with the shock terms (which are the only terms that affect the natural rate in the model without capital). In addition, in the model with capital we have derived two expressions relating the model shocks and natural rate terms (either 34 and 35 or 36 and 37). We shall see in section 5 that both of these equations will be needed in order to simplify our expression for the utility-based welfare criterion. ${ }^{4}$

\section{The Second-Order Approximation to the Utility Function}

We construct the second-order approximation to the overall utility function (equation 4) by deriving a second-order approximation to the within-period utility function,

$$
\frac{1}{1-\sigma}\left(C_{t}\right)^{1-\sigma} e^{a \chi_{t}^{c}}-\frac{1}{1+s}\left(H_{t}\right)^{1+s} e^{b \chi_{t}^{h}}
$$

Since within-period utility is additively separable between consumption and hours, we can consider the second-order approximations to each term in (38) separately. The second-order approximation to the consumption portion of within-period utility is given by:

$$
\begin{aligned}
\frac{1}{1-\sigma}\left(C_{t}\right)^{1-\sigma} e^{a \chi_{t}^{c}}= & U\left(C_{t}, \chi_{t}^{c}\right) \\
= & U\left(C_{*}, 0\right)+U_{C}\left(C_{*}, 0\right)\left(C_{t}-C_{*}\right)+U_{\chi^{c}}\left(C_{*}, 0\right) \chi_{t}^{c} \\
& +\frac{1}{2}\left(U_{C, C}\left(C_{*}, 0\right)\left(C_{t}-C_{*}\right)^{2}+U_{\chi^{c}, \chi^{c}}\left(C_{*}, 0\right)\left(\chi_{t}^{c}\right)^{2}+2 U_{C, \chi^{c}}\left(C_{*}, 0\right)\left(C_{t}-C_{*}\right) \chi_{t}^{c}\right) \\
= & \frac{1}{1-\sigma} C_{*}^{1-\sigma}+C_{*}^{1-\sigma}\left(c_{t}+\frac{1}{2} c_{t}^{2}\right)+\frac{1}{1-\sigma} C_{*}^{1-\sigma} a^{\prime} \chi_{t}^{c} \\
& +\frac{1}{2}\left(-\sigma C_{*}^{1-\sigma} c_{t}^{2}+\frac{1}{1-\sigma} C_{*}^{1-\sigma} \chi_{t}^{c \prime} a a^{\prime} \chi_{t}^{c}+2 C_{*}^{1-\sigma} c_{t} a^{\prime} \chi_{t}^{c}\right)+\mathcal{O}\left(\|\xi\|^{3}\right),
\end{aligned}
$$

\footnotetext{
${ }^{4}$ In the model without endogenous capital accumulation, only the first of these equations is needed to define the (considerably simplified) natural rate of output, $y_{t}^{n}=\left[\frac{s+\alpha}{1-\alpha}+\frac{\sigma}{c}\right]^{-1}\left(a \chi_{t}^{c}-b \chi_{t}^{h}+\frac{1+s}{1-\alpha} x_{t}\right)$; the second equation merely gives us the natural rate of interest that corresponds to the natural rate of output.
} 
where $c_{t}$ denotes the log-deviation of consumption from its steady-state value (an expression for which was obtained in section 2). Likewise, the second-order approximation to the hours component of within-period utility is given by:

$$
\begin{aligned}
\frac{1}{1+s}\left(H_{t}\right)^{1+s} e^{b \chi_{t}^{h}}= & V\left(H_{t}, \chi_{t}^{h}\right) \\
= & V\left(H_{*}, 0\right)+V_{H}\left(H_{*}, 0\right)\left(H_{t}-H_{*}\right)+V_{\chi^{h}}\left(H_{*}, 0\right) \chi_{t}^{h} \\
& +\frac{1}{2}\left(V_{H, H}\left(H_{*}, 0\right)\left(H_{t}-H_{*}\right)^{2}+V_{\chi^{h}, \chi^{h}}\left(H_{*}, 0\right)\left(\chi_{t}^{h}\right)^{2}+2 V_{H, \chi^{h}}\left(H_{*}, 0\right)\left(H_{t}-H_{*}\right) \chi_{t}^{h}\right) \\
= & \frac{1}{1+s} H_{*}^{1+s}+H_{*}^{1+s}\left(h_{t}+\frac{1}{2} h_{*}^{2}\right)+\frac{1}{1+s} H_{*}^{1+s} b^{\prime} \chi_{t}^{h} \\
& +\frac{1}{2}\left(s H_{*}^{1+s} h_{t}^{2}+\frac{1}{1+s} H_{*}^{1+s} \chi_{t}^{h^{\prime}} b b^{\prime} \chi_{t}^{h}+2 H_{*}^{1+s} h_{t} b^{\prime} \chi_{t}^{h}\right)+\mathcal{O}\left(\|\xi\|^{3}\right) .
\end{aligned}
$$

We would like our second-order approximation to the utility function to be expressed in terms of output and capital only. We must therefore eliminate all the terms involving $c_{t}$ and $h_{t}$ from equations (39) and (40).

To eliminate $\left(c_{t}+\frac{1}{2} c_{t}^{2}\right)$ from equation (39), we take the second-order approximation to the market-clearing condition (equation 19) combined with the second-order approximation to the capital evolution equation (3). Together, these two equations imply that

$c_{t}+\frac{1}{2} c_{t}^{2}=\frac{1}{\mathrm{c}}\left(y_{t}+\frac{1}{2} y_{t}^{2}\right)-\frac{1-\mathrm{c}}{\mathrm{c}} \cdot \frac{1}{\delta}\left(k_{t+1}+\frac{1}{2} k_{t+1}^{2}\right)+\frac{1-\mathrm{c}}{\mathrm{c}} \cdot \frac{1-\delta}{\delta}\left(k_{t}+\frac{1}{2} k_{t}^{2}\right)+\mathcal{O}\left(\|\xi\|^{3}\right)$.

Squaring the above equation provides us with an expression with which to replace $c_{t}^{2}$ in equation (39), while multiplying the above equation through by $a^{\prime} \chi_{t}^{c}$ gives us an expression with which to replace $c_{t} a^{\prime} \chi_{t}^{c}$. These are given by:

$$
\begin{aligned}
c_{t}^{2}= & \left(\frac{1}{\mathrm{c}}\right)^{2} y_{t}^{2}+\left(\frac{1-\mathrm{c}}{\mathrm{c}} \cdot \frac{1}{\delta}\right)^{2} k_{t+1}^{2}+\left(\frac{1-\mathrm{c}}{\mathrm{c}} \cdot \frac{1-\delta}{\delta}\right)^{2} k_{t}^{2} \\
& +2 \frac{1}{\mathrm{c}} \cdot \frac{1-\mathrm{c}}{\mathrm{c}} \cdot \frac{1-\delta}{\delta} y_{t} k_{t}-2 \frac{1}{\mathrm{c}} \cdot \frac{1-\mathrm{c}}{\mathrm{c}} \cdot \frac{1}{\delta} y_{t} k_{t+1}-2\left(\frac{1-\mathrm{c}}{\mathrm{c}}\right)^{2} \frac{1-\delta}{\delta} \cdot \frac{1}{\delta} k_{t} k_{t+1}+\mathcal{O}\left(\|\xi\|^{3}\right), \text { and } \\
c_{t} a^{\prime} \chi_{t}^{c} & =\frac{1}{\mathrm{c}} y_{t} a^{\prime} \chi_{t}^{c}-\frac{1-\mathrm{c}}{\mathrm{c}} \cdot \frac{1}{\delta} k_{t+1} a^{\prime} \chi_{t}^{c}+\frac{1-\mathrm{c}}{\mathrm{c}} \cdot \frac{1-\delta}{\delta} k_{t} a^{\prime} \chi_{t}^{c}+\mathcal{O}\left(\|\xi\|^{3}\right) .
\end{aligned}
$$

To eliminate the $h_{t}$ terms from equation (40), we first re-write firm $z$ 's production function (equation 2) as:

$$
Y_{t}(z)=X_{t}\left(\frac{K_{t}(z)}{H_{t}(z)}\right)^{\alpha} H_{t}(z)=X_{t}\left(\frac{K_{t}}{H_{t}}\right)^{\alpha} H_{t}(z)
$$

where the second equality comes from the fact that all firms face the same factor prices. Defining $H_{t}$ as $\int_{0}^{1} H_{t}(x) d x$, we write:

$$
H_{t}=\int_{0}^{1} H_{t}(x) d x=\left(\frac{H_{t}}{K_{t}}\right)^{\alpha} \frac{1}{X_{t}} \int_{0}^{1} Y_{t}(x) d x
$$


which we can re-arrange to yield

$$
H_{t}=\left(\frac{1}{K_{t}}\right)^{\frac{\alpha}{1-\alpha}}\left(\frac{1}{X_{t}}\right)^{\frac{1}{1-\alpha}}\left(\int_{0}^{1} Y_{t}(x) d x\right)^{\frac{1}{1-\alpha}} .
$$

To eliminate $\left(h_{t}+\frac{1}{2} h_{t}^{2}\right)$ from equation (40), we take the second-order approximation to equation (41), that is,

$$
\begin{aligned}
h_{t}+\frac{1}{2} h_{t}^{2}= & \frac{-\alpha}{1-\alpha}\left(k_{t}+\frac{1}{2} k_{t}^{2}\right)+\frac{1}{1-\alpha}\left(y_{t}+\frac{1}{2} y_{t}^{2}\right)-\frac{1}{1-\alpha}\left(x_{t}+\frac{1}{2} x_{t}^{2}\right)+\frac{1}{2} \cdot \frac{1}{1-\alpha}\left(\frac{1}{\theta}+\frac{\alpha}{1-\alpha}\right) \operatorname{var}_{z} y_{t}(z) \\
& +\frac{1}{2} \cdot \frac{\alpha}{1-\alpha} \cdot \frac{1}{1-\alpha} k_{t}^{2}+\frac{1}{2} \cdot \frac{\alpha}{1-\alpha} \cdot \frac{1}{1-\alpha} y_{t}^{2}-\frac{1}{2} \cdot \frac{1}{1-\alpha} \cdot \frac{2-\alpha}{1-\alpha} x_{t}^{2} \\
& -\frac{\alpha}{1-\alpha} \cdot \frac{1}{1-\alpha} y_{t} k_{t}+\frac{1}{1-\alpha} \cdot \frac{\alpha}{1-\alpha} x_{t} k_{t}-\left(\frac{1}{1-\alpha}\right)^{2} x_{t} y_{t}+\mathcal{O}\left(\|\xi\|^{3}\right) .
\end{aligned}
$$

Squaring the above equation provides us with an expression with which to replace $h_{t}^{2}$ in equation (40), while multiplying the above equation through by $b^{\prime} \chi_{t}^{h}$ yields an expression with which we can replace $h_{t} b^{\prime} \chi_{t}^{h}$. These expressions are:

$$
\begin{aligned}
h_{t}^{2} & =\left(\frac{\alpha}{1-\alpha}\right)^{2} k_{t}^{2}+\left(\frac{1}{1-\alpha}\right)^{2} y_{t}^{2}-2 \frac{\alpha}{1-\alpha} \cdot \frac{1}{1-\alpha} y_{t} k_{t}+\mathcal{O}\left(\|\xi\|^{3}\right) \text { and } \\
h_{t} b^{\prime} \chi_{t}^{h} & =\frac{-\alpha}{1-\alpha} k_{t} b^{\prime} \chi_{t}^{h}+\frac{1}{1-\alpha} y_{t} b^{\prime} \chi_{t}^{h}+\mathcal{O}\left(\|\xi\|^{3}\right) .
\end{aligned}
$$

The second-order approximation to the consumption term in the within-period utility function — with consumption replaced by output and capital —is:

$$
\begin{aligned}
& \frac{1}{1-\sigma}\left(C_{t}\right)^{1-\sigma} e^{a \chi_{t}^{c}} \\
= & \frac{C_{*}^{1-\sigma}}{\mathrm{c}} y_{t}-\frac{C_{*}^{1-\sigma}}{\mathrm{c}} \cdot \frac{1-\mathrm{c}}{\delta} k_{t+1}+\frac{C_{*}^{1-\sigma}}{\mathrm{c}} \cdot \frac{1-\mathrm{c}}{\delta}(1-\delta) k_{t}+\frac{1}{2} \cdot \frac{C_{*}^{1-\sigma}}{\mathrm{c}}\left(1-\frac{\sigma}{\mathrm{c}}\right) y_{t}^{2} \\
& -\frac{1}{2} \cdot \frac{C_{*}^{1-\sigma}}{\mathrm{c}} \cdot \frac{1-\mathrm{c}}{\delta}\left(1+\frac{\sigma}{\mathrm{c}} \cdot \frac{1-\mathrm{c}}{\delta}\right) k_{t+1}^{2}+\frac{1}{2} \cdot \frac{C_{*}^{1-\sigma}}{\mathrm{c}} \cdot \frac{1-\mathrm{c}}{\delta}(1-\delta)\left(1-\frac{\sigma}{\mathrm{c}} \cdot \frac{1-\mathrm{c}}{\delta} \cdot(1-\delta)\right) k_{t}^{2} \\
& -\frac{C_{*}^{1-\sigma}}{\mathrm{c}} \cdot \frac{\sigma}{\mathrm{c}} \cdot \frac{1-\mathrm{c}}{\delta}(1-\delta) y_{t} k_{t}+\frac{C_{*}^{1-\sigma}}{\mathrm{c}} \cdot \frac{\sigma}{\mathrm{c}} \cdot \frac{1-\mathrm{c}}{\delta} y_{t} k_{t+1}+\frac{C_{*}^{1-\sigma}}{\mathrm{c}} \cdot \frac{\sigma}{\mathrm{c}}\left(\frac{1-\mathrm{c}}{\delta}\right)^{2}(1-\delta) k_{t} k_{t+1} . \\
+ & \frac{C_{*}^{1-\sigma}}{\mathrm{c}} y_{t} a^{\prime} \chi_{t}^{c}-\frac{C_{*}^{1-\sigma}}{\mathrm{c}} \cdot \frac{1-\mathrm{c}}{\delta} k_{t+1} a^{\prime} \chi_{t}^{c}+\frac{C_{*}^{1-\sigma}}{\mathrm{c}} \cdot \frac{1-\mathrm{c}}{\delta}(1-\delta) k_{t} a^{\prime} \chi_{t}^{c}+\text { t.i.p. }+\mathcal{O}\left(\|\xi\|^{3}\right),
\end{aligned}
$$

where "t.i.p." denotes "terms independent of policy" (specifically, steady-state values and the variances of the stochastic terms).

Next, the second-order approximation to the hours term in the within-period utility function — with hours replaced by output and capital—is:

$$
\frac{1}{1+s}\left(H_{t}\right)^{1+s} e^{b \chi_{t}^{h}}
$$




$$
\begin{aligned}
& =\frac{H_{*}^{1+s}}{1-\alpha}(-\alpha) k_{t}+\frac{H_{*}^{1+s}}{1-\alpha} y_{t}+\frac{1}{2} \cdot \frac{H_{*}^{1+s}}{1-\alpha} \cdot \frac{\alpha}{1-\alpha} \alpha(1+s) k_{t}^{2}+\frac{1}{2} \cdot \frac{H_{*}^{1+s}}{1-\alpha} \cdot \frac{1+s}{1-\alpha} y_{t}^{2}-\frac{H_{*}^{1+s}}{1-\alpha} \cdot \frac{\alpha(1+s)}{1-\alpha} k_{t} y_{t} \\
& -\frac{H_{*}^{1+s}}{1-\alpha} \alpha k_{t} b^{\prime} \chi_{t}^{h}+\frac{H_{*}^{1+s}}{1-\alpha} y_{t} b^{\prime} \chi_{t}^{h}+\frac{H_{*}^{1+s}}{1-\alpha} \cdot \frac{\alpha(1+s)}{1-\alpha} k_{t} x_{t}-\frac{H_{*}^{1+s}}{1-\alpha} \cdot \frac{1+s}{1-\alpha} y_{t} x_{t} \\
& +\frac{1}{2} \cdot \frac{H_{*}^{1+s}}{1-\alpha} \cdot \frac{1}{1-\alpha}\left(\frac{1}{\theta}+\frac{\alpha}{1-\alpha}\right) \operatorname{var}_{z} y_{t}(z)+\text { t.i.p. }+\mathcal{O}\left(\|\xi\|^{3}\right) .
\end{aligned}
$$

We now combine equations (42) and (43) in order to obtain a second-order approximation for the within-period utility function (equation 38). In doing so, we group terms according to whether they are first-order terms, second-order terms in the model's variables, and secondorder cross-products between the model variables and shocks. Note that we can take the multiplicative terms $\frac{C_{*}^{1-\sigma}}{\mathrm{c}}$ and $\frac{H_{*}^{1+s}}{1-\alpha}$, which we know are equal from equation (21), to the other side of the expression. We therefore write

$$
\begin{aligned}
& \left(\frac{1}{1-\sigma}\left(C_{t}\right)^{1-\sigma} e^{a \chi_{t}^{c}}-\frac{1}{1+s}\left(H_{t}\right)^{1+s} e^{b \chi_{t}^{h}}\right)\left(\frac{C_{*}^{1-\sigma}}{\mathrm{c}}\right)^{-1} \\
& =\underbrace{y_{t}-\frac{1-\mathrm{c}}{\delta} k_{t+1}+\frac{1-\mathrm{c}}{\delta}(1-\delta) k_{t}+\alpha k_{t}-y_{t}}_{\text {first-order terms }}+\frac{1}{2}\left(1-\frac{\sigma}{\mathrm{c}}\right) y_{t}^{2}+\frac{1}{2}\left(-\frac{1-\mathrm{c}}{\delta}+-\frac{\sigma}{\mathrm{c}}\left(\frac{1-\mathrm{c}}{\delta}\right)^{2}\right) k_{t+1}^{2} \\
& +\frac{1}{2}\left(\frac{1-\mathrm{c}}{\delta}(1-\delta)+-\frac{\sigma}{\mathrm{c}}\left(\frac{1-\mathrm{c}}{\delta}\right)^{2}(1-\delta)^{2}\right) k_{t}^{2}-\frac{\sigma}{\mathrm{c}} \cdot \frac{1-\mathrm{c}}{\delta}(1-\delta) y_{t} k_{t}+\frac{\sigma}{\mathrm{c}} \cdot \frac{1-\mathrm{c}}{\delta} y_{t} k_{t+1} \\
& +\frac{\sigma}{\mathrm{c}}\left(\frac{1-\mathrm{c}}{\delta}\right)^{2}(1-\delta) k_{t} k_{t+1}+\frac{1}{2} \cdot \frac{-\alpha}{1-\alpha} \alpha(1+s) k_{t}^{2}+\frac{1}{2} \cdot \frac{-(1+s)}{1-\alpha} y_{t}^{2}+\frac{\alpha(1+s)}{1-\alpha} k_{t} y_{t} \\
& +\underbrace{y_{t} a^{\prime} \chi_{t}^{c}-\frac{1-\mathrm{c}}{\delta} k_{t+1} a^{\prime} \chi_{t}^{c}+\frac{1-\mathrm{c}}{\delta}(1-\delta) k_{t} a^{\prime} \chi_{t}^{c}+\alpha k_{t} b^{\prime} \chi_{t}^{h}-y_{t} b^{\prime} \chi_{t}^{h}-\frac{\alpha(1+s)}{1-\alpha} k_{t} x_{t}+\frac{1+s}{1-\alpha} y_{t} x_{t}}_{\text {model-variable/shock cross-product terms }} \\
& -\frac{1}{2} \cdot \frac{1}{1-\alpha}\left(\frac{1}{\theta}+\frac{\alpha}{1-\alpha}\right) \operatorname{var}_{z} y_{t}(z)+\text { t.i.p. }+\mathcal{O}\left(\|\xi\|^{3}\right) .
\end{aligned}
$$

The obvious next step is to simplify equation (44); we do this in the next section. To effect the simplification, we note that the second-order approximation to the overall utility function (equation 4) will involve a (discounted) expression like equation (44) that corresponds to each term in the overall utility function.

\section{Simplifying the Approximate Utility Function}

We start by simplifying the within-period utility expression (44), which we do in two steps. First, we eliminate all first-order terms from the expression. Next, we simplify the cross 
products of the model variables and shock terms. This will then permit us to obtain an approximate expression for the overall utility function, which we will write in terms of both the Neiss-Nelson and Woodford natural rate definitions.

\subsection{Eliminating First-Order Terms from Within-Period Utility}

As simple inspection reveals, it is straightforward to eliminate output from the first-order terms in equation (44):

$$
y_{t}-\frac{1-\mathrm{c}}{\delta} k_{t+1}+\frac{1-\mathrm{c}}{\delta}(1-\delta) k_{t}+\alpha k_{t}-y_{t}=\frac{1-\mathrm{c}}{\delta} k_{t+1}+\frac{1-\mathrm{c}}{\delta}(1-\delta) k_{t}+\alpha k_{t} .
$$

Simplifying the remaining terms - which clearly arise only in a model with endogenous capital accumulation - is a little more complicated. First, we need to consider what $(1-c) / \delta$ equals. Since $\mathrm{c}=C_{*} / Y_{*}$ we know that $(1-\mathrm{c})=I_{*} / Y_{*}$, which we defined in equation (22). This implies that

$$
\frac{1-\mathrm{c}}{\delta}=\frac{\beta \alpha}{1-\beta(1-\delta)},
$$

which in turn implies that the remaining terms can be simplified as follows:

$$
\begin{aligned}
- & \frac{1-\mathrm{c}}{\delta} k_{t+1}+\frac{1-\mathrm{c}}{\delta}(1-\delta) k_{t}+\alpha k_{t} \\
& =\frac{-\beta \alpha}{1-\beta(1-\delta)} k_{t+1}+\frac{\beta \alpha}{1-\beta(1-\delta)}(1-\delta) k_{t}+\alpha k_{t} \\
& =\alpha\left(\frac{-\beta}{1-\beta(1-\delta)} k_{t+1}+\frac{\beta(1-\delta)}{1-\beta(1-\delta)} k_{t}+\frac{1-\beta(1-\delta)}{1-\beta(1-\delta)} k_{t}\right)=\alpha \frac{1}{1-\beta(1-\delta)}\left(k_{t}-\beta k_{t+1}\right) .
\end{aligned}
$$

Further simplification requires us to cancel terms across different periods. Recall that the second-order approximation to the overall utility function is given by the discounted sum of the second-order approximations to the within-period utility functions (that is, equation 44). We can therefore pull together all of the first-order terms that remain after simplification from each within-period utility function to obtain

$$
\frac{\alpha}{1-\beta(1-\delta)} E_{0}\left[\left(k_{0}-\beta k_{1}\right)+\beta\left(k_{1}-\beta k_{2}\right)+\cdots+\beta^{t}\left(k_{t}-\beta k_{t+1}\right)+\beta^{t}\left(k_{t+1}-\beta k_{t+2}\right)+\cdots\right],
$$

which when we cancel terms from different periods is just equal to:

$$
\frac{\alpha}{1-\beta(1-\delta)} k_{0}
$$

In other words, all first-order terms disappear except for a term in $k_{0}$ (the initial capital stock), which is assumed to be fixed and independent of policy. 


\subsection{Simplifying the Cross-Product Terms}

The second-order cross-products of the model variables and shocks in equation (44) are:

$$
\begin{gathered}
y_{t} a^{\prime} \chi_{t}^{c}-\frac{1-\mathrm{c}}{\delta} k_{t+1} a^{\prime} \chi_{t}^{c}+\frac{1-\mathrm{c}}{\delta}(1-\delta) k_{t} a^{\prime} \chi_{t}^{c}+\alpha k_{t} b^{\prime} \chi_{t}^{h}-y_{t} b^{\prime} \chi_{t}^{h}-\frac{\alpha(1+s)}{1-\alpha} k_{t} x_{t}+\frac{1+s}{1-\alpha} y_{t} x_{t} \\
=y_{t}\left[a^{\prime} \chi_{t}^{c}-b^{\prime} \chi_{t}^{h}+\frac{1+s}{1-\alpha} x_{t}\right]-\frac{1-\mathrm{c}}{\delta} k_{t+1} a^{\prime} \chi_{t}^{c}+\frac{1-\mathrm{c}}{\delta}(1-\delta) k_{t} a^{\prime} \chi_{t}^{c}+\alpha k_{t} b^{\prime} \chi_{t}^{h}-\frac{\alpha(1+s)}{1-\alpha} k_{t} x_{t} .
\end{gathered}
$$

Note that we can substitute in for $(1-\mathrm{c}) / \delta$ using equation (45) to obtain:

$$
\begin{aligned}
y_{t}\left[a^{\prime} \chi_{t}^{c}-b^{\prime} \chi_{t}^{h}+\frac{1+s}{1-\alpha} x_{t}\right] & -\frac{\beta \alpha}{1-\beta(1-\delta)} k_{t+1} a^{\prime} \chi_{t}^{c} \\
& +\frac{\beta \alpha(1-\delta)}{1-\beta(1-\delta)} k_{t} a^{\prime} \chi_{t}^{c}+\alpha \frac{1-\beta(1-\delta)}{1-\beta(1-\delta)} k_{t} b^{\prime} \chi_{t}^{h}-\frac{\alpha(1+s)}{1-\alpha} k_{t} x_{t} \\
=y_{t}\left[a^{\prime} \chi_{t}^{c}-b^{\prime} \chi_{t}^{h}+\frac{1+s}{1-\alpha} x_{t}\right] & +\frac{\beta \alpha(1-\delta)}{1-\beta(1-\delta)} k_{t}\left[a^{\prime} \chi_{t}^{c}-b^{\prime} \chi_{t}^{h}\right] \\
& -\frac{\alpha(1+s)}{1-\alpha} k_{t} x_{t}+\frac{\alpha}{1-\beta(1-\delta)}\left[k_{t} b^{\prime} \chi_{t}^{h}-\beta k_{t+1} a^{\prime} \chi_{t}^{c}\right] .
\end{aligned}
$$

We also have an expression for $\left[a^{\prime} \chi_{t}^{c}-b^{\prime} \chi_{t}^{h}+\frac{1+s}{1-\alpha} x_{t}\right]$ from equation (34) or (36) (depending on whether we use Neiss and Nelson's or Woodford's definition of the natural rate of output). We do not have any expressions for the remaining shock terms, and so will need to perform additional manipulations in order to obtain a form that will allow us to replace these shock terms with variables from the flexible-price model. To do this, we must take a stand on a definition of the natural rate of output. We will first consider the Neiss-Nelson definition, which - given its symmetry - is easier to implement; next, we will examine the Woodford definition, which yields a more intuitive concept for potential output.

\subsubsection{Derivation under the Neiss-Nelson Natural Rate Definition}

We focus on the final term $\left[k_{t} b^{\prime} \chi_{t}^{h}-\beta k_{t+1} a^{\prime} \chi_{t}^{c}\right]$ in equation (46), and replace $a^{\prime} \chi_{t}^{c}$ with what it equals when Neiss and Nelson's (2003) definition of the natural rate of output is employed (c.f. equation 35). Specifically,

$$
\begin{aligned}
& {\left[k_{t} b^{\prime} \chi_{t}^{h}-\beta k_{t+1} a^{\prime} \chi_{t}^{c}\right]} \\
& \begin{array}{l}
\underbrace{k_{t} b^{\prime} \chi_{t}^{h}}_{B(t)} \underbrace{-\beta k_{t+1} a^{\prime} \chi_{t+1}^{c}}_{A(t)}-(1-\beta(1-\delta))\left(\beta k_{t+1} y_{t+1}^{n}-\beta k_{t+1} k_{t+1}^{n}\right)+\frac{\sigma}{c}\left(\beta k_{t+1} y_{t+1}^{n}-\beta k_{t+1} y_{t}^{n}\right) \\
\quad-\frac{\sigma}{c} \cdot \frac{1-\mathrm{c}}{\delta}\left(\beta k_{t+1} k_{t+2}^{n}-\beta k_{t+1} k_{t+1}^{n}\right)+\frac{\sigma}{\mathrm{c}} \cdot \frac{1-\mathrm{c}}{\delta}(1-\delta)\left(\beta k_{t+1} k_{t+1}^{n}-\beta k_{t+1} k_{t}^{n}\right) .
\end{array}
\end{aligned}
$$


A similar expression appears in the second-order approximation to the $t+1$ within-period utility function; namely,

$$
\begin{aligned}
& {\left[k_{t} b^{\prime} \chi_{t+1}^{h}-\beta k_{t+1} a^{\prime} \chi_{t+1}^{c}\right] } \\
&=\underbrace{\beta k_{t+1} b^{\prime} \chi_{t+1}^{h}-\beta^{2} k_{t+2} a^{\prime} \chi_{t+2}^{c}}_{B(t+1)}-(1-\beta(1-\delta))\left(\beta^{2} k_{t+2} y_{t+2}^{n}-\beta^{2} k_{t+2} k_{t+2}^{n}\right) \\
&+\frac{\sigma}{\mathrm{c}}\left(\beta^{2} k_{t+2} y_{t+2}^{n}-\beta^{2} k_{t+2} y_{t+1}^{n}\right) \\
&-\frac{\sigma}{\mathrm{c}} \cdot \frac{1-\mathrm{c}}{\delta}\left(\beta^{2} k_{t+2} k_{t+3}^{n}-\beta^{2} k_{t+2} k_{t+2}^{n}\right)+\frac{\sigma}{\mathrm{c}} \cdot \frac{1-\mathrm{c}}{\delta}(1-\delta)\left(\beta^{2} k_{t+2} k_{t+2}^{n}-\beta^{2} k_{t+2} k_{t+1}^{n}\right) .
\end{aligned}
$$

We can group $A$ and $B$ from the two different periods (e.g. $A(t)$ and $B(t+1)$ or $A(t-1)$ and $B(t))$, which yields a term equal to $-\beta k_{t+1}\left[a^{\prime} \chi_{t+1}^{c}-b^{\prime} \chi_{t+1}^{h}\right]$ in period $t+1$ and a term equal to $-k_{t}\left[a^{\prime} \chi_{t}^{c}-b^{\prime} \chi_{t}^{h}\right]$ in period $t$. Hence, we have:

$$
\begin{aligned}
& {\left[k_{t} b^{\prime} \chi_{t}^{h}-\beta k_{t+1} a^{\prime} \chi_{t}^{c}\right]} \\
& \begin{aligned}
=\underbrace{k_{t} b^{\prime} \chi_{t}^{h}-k_{t} a^{\prime} \chi_{t}^{c}}_{B(t)}-(1-\beta(1-\delta))\left(\beta k_{t+1} y_{t+1}^{n}-\beta k_{t+1} k_{t+1}^{n}\right)+\frac{\sigma}{c}\left(\beta k_{t+1} y_{t+1}^{n}-\beta k_{t+1} y_{t}^{n}\right) \\
\quad-\frac{\sigma}{\mathrm{c}} \cdot \frac{1-\mathrm{c}}{\delta}\left(\beta k_{t+1} k_{t+2}^{n}-\beta k_{t+1} k_{t+1}^{n}\right)+\frac{\sigma}{\mathrm{c}} \cdot \frac{1-\mathrm{c}}{\delta}(1-\delta)\left(\beta k_{t+1} k_{t+1}^{n}-\beta k_{t+1} k_{t}^{n}\right),
\end{aligned}
\end{aligned}
$$

which we can use to replace $\left[k_{t} b^{\prime} \chi_{t}^{h}-\beta k_{t+1} a^{\prime} \chi_{t}^{c}\right]$ in equation (46). Note that, because we are moving the $A$ term from an earlier period (for example, $t-1$ ) to a later period (in this case, time $t$ ), we will have to add the term $k_{0} a^{\prime} \chi_{t}^{c}$ in the initial period; otherwise, the within-period loss function will not be the same for all periods. For considering the problem of maximizing household welfare, this is immaterial because this term is independent of policy; however, it does mean that the expression that we will be working with is only proportional to the second-order approximation. In what follows, I ignore any such additive scaling terms.

The preceding substitutions imply that equation (46) - that is the cross-products of the model variables and shocks - are:

$$
\begin{aligned}
& y_{t}\left[a^{\prime} \chi_{t}^{c}-b^{\prime} \chi_{t}^{h}+\frac{1+s}{1-\alpha} x_{t}\right]+\frac{\beta \alpha(1-\delta)}{1-\beta(1-\delta)} k_{t}\left[a^{\prime} \chi_{t}^{c}-b^{\prime} \chi_{t}^{h}\right]-\frac{\alpha(1+s)}{1-\alpha} k_{t} x_{t}+\frac{-\alpha}{1-\beta(1-\delta)} k_{t}\left[a^{\prime} \chi_{t}^{c}-b^{\prime} \chi_{t}^{h}\right] \\
& -\frac{\alpha}{1-\beta(1-\delta)}(1-\beta(1-\delta))\left(\beta k_{t+1} y_{t+1}^{n}-\beta k_{t+1} k_{t+1}^{n}\right)+\frac{\alpha}{1-\beta(1-\delta)} \cdot \frac{\sigma}{\mathrm{c}}\left(\beta k_{t+1} y_{t+1}^{n}-\beta k_{t+1} y_{t}^{n}\right) \\
& -\frac{\alpha}{1-\beta(1-\delta)} \cdot \frac{\sigma}{\mathrm{c}} \cdot \frac{1-\mathrm{c}}{\delta}\left(\beta k_{t+1} k_{t+2}^{n}-\beta k_{t+1} k_{t+1}^{n}\right)+\frac{\alpha}{1-\beta(1-\delta)} \cdot \frac{\sigma}{\mathrm{c}} \cdot \frac{1-\mathrm{c}}{\delta}(1-\delta)\left(\beta k_{t+1} k_{t+1}^{n}-\beta k_{t+1} k_{t}^{n}\right) . \\
& =y_{t}\left[a^{\prime} \chi_{t}^{c}-b^{\prime} \chi_{t}^{h}+\frac{1+s}{1-\alpha} x_{t}\right]-\alpha k_{t}\left[a^{\prime} \chi_{t}^{c}-b^{\prime} \chi_{t}^{h}-\frac{1+s}{1-\alpha} x_{t}\right]-\alpha\left(\beta k_{t+1} y_{t+1}^{n}-\beta k_{t+1} k_{t+1}^{n}\right)
\end{aligned}
$$




$$
\begin{aligned}
& +\frac{\alpha}{1-\beta(1-\delta)} \cdot \frac{\sigma}{\mathrm{c}}\left(\beta k_{t+1} y_{t+1}^{n}-\beta k_{t+1} y_{t}^{n}\right)-\frac{\alpha}{1-\beta(1-\delta)} \cdot \frac{\sigma}{\mathrm{c}} \cdot \frac{1-\mathrm{c}}{\delta}\left(\beta k_{t+1} k_{t+2}^{n}-\beta k_{t+1} k_{t+1}^{n}\right) \\
& +\frac{\alpha}{1-\beta(1-\delta)} \cdot \frac{\sigma}{\mathrm{c}} \cdot \frac{1-\mathrm{c}}{\delta}(1-\delta)\left(\beta k_{t+1} k_{t+1}^{n}-\beta k_{t+1} k_{t}^{n}\right) .
\end{aligned}
$$

We already have an expression for $\left[a^{\prime} \chi_{t}^{c}-b^{\prime} \chi_{t}^{h}+\frac{1+s}{1-\alpha} x_{t}\right]$ from equation (34). Substituting this expression into (49) would then eliminate all of the cross products between the model variables and shocks, thereby leaving us with a set of cross products between the model variables and natural rate terms. Specifically, we obtain an expression for these terms that is given by:

$$
\begin{aligned}
& {\left[\frac{s+\alpha}{1-\alpha}+\frac{\sigma}{\mathrm{c}}\right] y_{t} y_{t}^{n}+\left[-\frac{\alpha(1+s)}{1-\alpha}+\frac{\sigma}{\mathrm{c}} \cdot \frac{1-\mathrm{c}}{\delta}(1-\delta)\right] y_{t} k_{t}^{n}+\left[-\frac{\sigma}{\mathrm{c}} \cdot \frac{1-\mathrm{c}}{\delta}\right] y_{t} k_{t+1}^{n}} \\
& +\left[\frac{\beta \alpha(1-\delta)}{1-\beta(1-\delta)}\right]\left[\frac{s+\alpha}{1-\alpha}+\frac{\sigma}{\mathrm{c}}\right] k_{t} y_{t}^{n}+\left[\frac{\beta \alpha(1-\delta)}{1-\beta(1-\delta)}\right]\left[-\frac{\alpha(1+s)}{1-\alpha}+\frac{\sigma}{\mathrm{c}} \cdot \frac{1-\mathrm{c}}{\delta}(1-\delta)\right] k_{t} k_{t}^{n} \\
& +\left[\frac{\beta \alpha(1-\delta)}{1-\beta(1-\delta)}\right]\left[-\frac{\sigma}{\mathrm{c}} \cdot \frac{1-\mathrm{c}}{\delta}\right] k_{t} k_{t+1}^{n}+\left[\frac{\alpha}{1-\beta(1-\delta)}\right]\left[-\frac{s+\alpha}{1-\alpha}-\frac{\sigma}{\mathrm{c}}\right] k_{t} y_{t}^{n} \\
& +\left[\frac{\alpha}{1-\beta(1-\delta)}\right]\left[\frac{\alpha(1+s)}{1-\alpha}-\frac{\sigma}{\mathrm{c}} \cdot \frac{1-\mathrm{c}}{\delta}(1-\delta)\right] k_{t} k_{t}^{n}+\left[\frac{\alpha}{1-\beta(1-\delta)}\right]\left[\frac{\sigma}{\mathrm{c}} \cdot \frac{1-\mathrm{c}}{\delta}\right] k_{t} k_{t+1}^{n} \\
& -\alpha \beta\left(k_{t+1} y_{t+1}^{n}-k_{t+1} k_{t+1}^{n}\right)+\left[\frac{\alpha \beta}{1-\beta(1-\delta)}\right]\left[\frac{\sigma}{\mathrm{c}}\right] k_{t+1} y_{t+1}^{n}+\left[\frac{\alpha \beta}{1-\beta(1-\delta)}\right]\left[-\frac{\sigma}{\mathrm{c}}\right] k_{t+1} y_{t}^{n} \\
& +\left[\frac{\alpha \beta}{1-\beta(1-\delta)}\right]\left[-\frac{\sigma}{\mathrm{c}} \cdot \frac{1-\mathrm{c}}{\delta}\right] k_{t+1} k_{t+2}^{n}+\left[\frac{\alpha \beta}{1-\beta(1-\delta)}\right]\left[\frac{\sigma}{\mathrm{c}} \cdot \frac{1-\mathrm{c}}{\delta}\right] k_{t+1} k_{t+1}^{n} \\
& +\left[\frac{\alpha \beta}{1-\beta(1-\delta)}\right]\left[\frac{\sigma}{\mathrm{c}} \cdot \frac{1-\mathrm{c}}{\delta}(1-\delta)\right] k_{t+1} k_{t+1}^{n}+\left[\frac{\alpha \beta}{1-\beta(1-\delta)}\right]\left[-\frac{\sigma}{\mathrm{c}} \cdot \frac{1-\mathrm{c}}{\delta}(1-\delta)\right] k_{t+1} k_{t}^{n} \cdot(50)
\end{aligned}
$$

In considering the second-order approximation to the utility function, this would replace the cross-products between the model variables and shocks in equation (44) when the natural rate of output corresponds to Neiss and Nelson's definition.

\subsubsection{Derivation under the Woodford Natural Rate Definition}

We can follow essentially the same steps when we use Woodford's definition of the natural rate of output; however, when we replace $a^{\prime} \chi_{t}^{c}$ with its value from the flexible-price model in the $\left[k_{t} b^{\prime} \chi_{t}^{h}-\beta k_{t+1} a^{\prime} \chi_{t}^{c}\right]$ term of equation (46), we will now use equation (37), rather than equation (35). Thus, with the Woodford definition of the natural rate of output, we obtain that

$$
\left[k_{t} b^{\prime} \chi_{t}^{h}-\beta k_{t+1} a^{\prime} \chi_{t}^{c}\right]
$$




$$
\begin{array}{r}
=\underbrace{k_{t} b^{\prime} \chi_{t}^{h}-k_{t} a^{\prime} \chi_{t}^{c}}_{B(t)}-(1-\beta(1-\delta))\left(\beta k_{t+1} y_{t+1}^{n}-\beta k_{t+1} k_{t+1}^{n}\right)+\frac{\sigma}{\mathrm{c}}\left(\beta k_{t+1} y_{t+1}^{n}-\beta k_{t+1} y_{t}^{n}\right) \\
-\frac{\sigma}{\mathrm{c}} \cdot \frac{1-\mathrm{c}}{\delta}\left(\beta k_{t+1} k_{t+2}^{n}-\beta k_{t+1} k_{t+1}^{n}\right)+\frac{\sigma}{\mathrm{c}} \cdot \frac{1-\mathrm{c}}{\delta}(1-\delta)\left(\beta k_{t+1} k_{t+1}^{n}-\beta k_{t+1} k_{t}\right) .
\end{array}
$$

We use this to replace $\left[k_{t} b^{\prime} \chi_{t}^{h}-\beta k_{t+1} a^{\prime} \chi_{t}^{c}\right]$ in equation (46), which implies that when we employ Woodford's definition of the natural rate, the cross-product terms for the model variables and shocks become:

$$
\begin{aligned}
& y_{t}\left[a^{\prime} \chi_{t}^{c}-b^{\prime} \chi_{t}^{h}+\frac{1+s}{1-\alpha} x_{t}\right]-\alpha k_{t}\left[a^{\prime} \chi_{t}^{c}-b^{\prime} \chi_{t}^{h}-\frac{1+s}{1-\alpha} x_{t}\right]-\alpha\left(\beta k_{t+1} y_{t+1}^{n}-\beta k_{t+1} k_{t+1}^{n}\right) \\
& +\frac{\alpha}{1-\beta(1-\delta)} \cdot \frac{\sigma}{\mathrm{c}}\left(\beta k_{t+1} y_{t+1}^{n}-\beta k_{t+1} y_{t}^{n}\right)-\frac{\alpha}{1-\beta(1-\delta)} \cdot \frac{\sigma}{\mathrm{c}} \cdot \frac{1-\mathrm{c}}{\delta}\left(\beta k_{t+1} k_{t+2}^{n}-\beta k_{t+1} k_{t+1}^{n}\right) \\
& \quad+\frac{\alpha}{1-\beta(1-\delta)} \frac{\sigma}{\mathrm{c}} \cdot \frac{1-\mathrm{c}}{\delta}(1-\delta)\left(\beta k_{t+1}^{2}-\beta k_{t+1} k_{t}\right) .
\end{aligned}
$$

We have an expression for $\left[a^{\prime} \chi_{t}^{c}-b^{\prime} \chi_{t}^{h}+\frac{1+s}{1-\alpha} x_{t}\right]$ which is now given by equation (36). Substituting this expression into equation (51) would then eliminate all cross-product terms in the variables and shocks, replacing them (as before) with cross products in the model variables and natural-rate terms. Specifically, we find that these equal:

$$
\begin{aligned}
& {\left[\frac{s+\alpha}{1-\alpha}+\frac{\sigma}{\mathrm{c}}\right] y_{t} y_{t}^{n}+\left[-\frac{\alpha(1+s)}{1-\alpha}+\frac{\sigma}{\mathrm{c}} \cdot \frac{1-\mathrm{c}}{\delta}(1-\delta)\right] y_{t} k_{t}+\left[-\frac{\sigma}{\mathrm{c}} \cdot \frac{1-\mathrm{c}}{\delta}\right] y_{t} k_{t+1}^{n}} \\
& +\left[\frac{\beta \alpha(1-\delta)}{1-\beta(1-\delta)}\right]\left[\frac{s+\alpha}{1-\alpha}+\frac{\sigma}{\mathrm{c}}\right] k_{t} y_{t}^{n}+\left[\frac{\beta \alpha(1-\delta)}{1-\beta(1-\delta)}\right]\left[-\frac{\alpha(1+s)}{1-\alpha}+\frac{\sigma}{\mathrm{c}} \cdot \frac{1-\mathrm{c}}{\delta}(1-\delta)\right] k_{t}^{2} \\
& +\left[\frac{\beta \alpha(1-\delta)}{1-\beta(1-\delta)}\right]\left[-\frac{\sigma}{\mathrm{c}} \cdot \frac{1-\mathrm{c}}{\delta}\right] k_{t} k_{t+1}^{n}+\left[\frac{\alpha}{1-\beta(1-\delta)}\right]\left[-\frac{s+\alpha}{1-\alpha}-\frac{\sigma}{\mathrm{c}}\right] k_{t} y_{t}^{n} \\
& +\left[\frac{\alpha}{1-\beta(1-\delta)}\right]\left[\frac{\alpha(1+s)}{1-\alpha}-\frac{\sigma}{\mathrm{c}} \cdot \frac{1-\mathrm{c}}{\delta}(1-\delta)\right] k_{t}^{2}+\left[\frac{\alpha}{1-\beta(1-\delta)}\right]\left[\frac{\sigma}{\mathrm{c}} \cdot \frac{1-\mathrm{c}}{\delta}\right] k_{t} k_{t+1}^{n} \\
& -\alpha \beta\left(k_{t+1} y_{t+1}^{n}-k_{t+1} k_{t+1}^{n}\right)+\left[\frac{\alpha \beta}{1-\beta(1-\delta)}\right]\left[\frac{\sigma}{\mathrm{c}}\right] k_{t+1} y_{t+1}^{n}+\left[\frac{\alpha \beta}{1-\beta(1-\delta)}\right]\left[-\frac{\sigma}{\mathrm{c}}\right] k_{t+1} y_{t}^{n} \\
& +\left[\frac{\alpha \beta}{1-\beta(1-\delta)}\right]\left[-\frac{\sigma}{\mathrm{c}} \cdot \frac{1-\mathrm{c}}{\delta}\right] k_{t+1} k_{t+2}^{n}+\left[\frac{\alpha \beta}{1-\beta(1-\delta)}\right]\left[\frac{\sigma}{\mathrm{c}} \cdot \frac{1-\mathrm{c}}{\delta}\right] k_{t+1} k_{t+1}^{n} \\
& +\left[\frac{\alpha \beta}{1-\beta(1-\delta)}\right]\left[\frac{\sigma}{\mathrm{c}} \cdot \frac{1-\mathrm{c}}{\delta}(1-\delta)\right] k_{t+1}^{2}+\left[\frac{\alpha \beta}{1-\beta(1-\delta)}\right]\left[-\frac{\sigma}{\mathrm{c}} \cdot \frac{1-\mathrm{c}}{\delta}(1-\delta)\right] k_{t+1} k_{t} .
\end{aligned}
$$

This replaces the cross-product terms in equation (44).

\subsection{The Approximation to the Overall Utility Function}

We now have everything that we require in order to construct the second-order approximation to the overall utility function. 


\subsubsection{Derivation Using the Neiss-Nelson Natural Rate}

Under the Neiss-Nelson natural rate definition, we can combine the second-order approximation to the within-period utility function (given by equation 44) with the model-variable/naturalrate cross product expressions outlined in section 5.2. This yields the following second-order approximation to the overall utility function:

$$
\begin{aligned}
& \left(\frac{C_{*}^{1-\sigma}}{\mathrm{c}}\right)^{-1} E_{0}\left[\sum_{t=0}^{\infty} \beta^{t}\left(\frac{1}{1-\sigma}\left(C_{t}\right)^{1-\sigma} e^{a \chi_{t}^{c}}-\frac{1}{1+s}\left(H_{t}\right)^{1+s} e^{b \chi_{t}^{h}}\right)\right] \\
& =E_{0}\left[\sum _ { t = 0 } ^ { \infty } \beta ^ { t } \left(\frac{1}{2}\left(1-\frac{\sigma}{\mathrm{c}}\right) y_{t}^{2}+\frac{1}{2}\left(-\frac{1-\mathrm{c}}{\delta}-\frac{\sigma}{\mathrm{c}}\left(\frac{1-\mathrm{c}}{\delta}\right)^{2}\right) k_{t+1}^{2}+\frac{1}{2}\left(\frac{1-\mathrm{c}}{\delta}(1-\delta)-\frac{\sigma}{\mathrm{c}}\left(\frac{1-\mathrm{c}}{\delta}\right)^{2}(1-\delta)^{2}\right) k_{t}^{2}\right.\right. \\
& -\frac{\sigma}{\mathrm{c}} \cdot \frac{1-\mathrm{c}}{\delta}(1-\delta) y_{t} k_{t}+\frac{\sigma}{\mathrm{c}} \cdot \frac{1-\mathrm{c}}{\delta} y_{t} k_{t+1}+\frac{\sigma}{\mathrm{c}}\left(\frac{1-\mathrm{c}}{\delta}\right)^{2}(1-\delta) k_{t} k_{t+1}+\frac{1}{2} \cdot \frac{-\alpha}{1-\alpha} \alpha(1+s) k_{t}^{2} \\
& +\frac{1}{2} \cdot \frac{-(1+s)}{1-\alpha} y_{t}^{2}+\frac{\alpha(1+s)}{1-\alpha} k_{t} y_{t}-\frac{1}{2} \cdot \frac{1}{1-\alpha}\left(\frac{1}{\theta}+\frac{\alpha}{1-\alpha}\right) \operatorname{var}_{z} y_{t}(z) \\
& +\left[\frac{s+\alpha}{1-\alpha}+\frac{\sigma}{\mathrm{c}}\right] y_{t} y_{t}^{n}+\left[-\frac{\alpha(1+s)}{1-\alpha}+\frac{\sigma}{\mathrm{c}} \cdot \frac{1-\mathrm{c}}{\delta}(1-\delta)\right] y_{t} k_{t}^{n}+\left[-\frac{\sigma}{\mathrm{c}} \cdot \frac{1-\mathrm{c}}{\delta}\right] y_{t} k_{t+1}^{n} \\
& +\left[\frac{\beta \alpha(1-\delta)}{1-\beta(1-\delta)}\right]\left[\frac{s+\alpha}{1-\alpha}+\frac{\sigma}{\mathrm{c}}\right] k_{t} y_{t}^{n}+\left[\frac{\beta \alpha(1-\delta)}{1-\beta(1-\delta)}\right]\left[-\frac{\alpha(1+s)}{1-\alpha}+\frac{\sigma}{\mathrm{c}} \cdot \frac{1-\mathrm{c}}{\delta}(1-\delta)\right] k_{t} k_{t}^{n} \\
& +\left[\frac{\beta \alpha(1-\delta)}{1-\beta(1-\delta)}\right]\left[-\frac{\sigma}{\mathrm{c}} \cdot \frac{1-\mathrm{c}}{\delta}\right] k_{t} k_{t+1}^{n}+\left[\frac{\alpha}{1-\beta(1-\delta)}\right]\left[-\frac{s+\alpha}{1-\alpha}-\frac{\sigma}{\mathrm{c}}\right] k_{t} y_{t}^{n} \\
& +\left[\frac{\alpha}{1-\beta(1-\delta)}\right]\left[\frac{\alpha(1+s)}{1-\alpha}-\frac{\sigma}{\mathrm{c}} \cdot \frac{1-\mathrm{c}}{\delta}(1-\delta)\right] k_{t} k_{t}^{n}+\left[\frac{\alpha}{1-\beta(1-\delta)}\right]\left[\frac{\sigma}{\mathrm{c}} \cdot \frac{1-\mathrm{c}}{\delta}\right] k_{t} k_{t+1}^{n} \\
& -\alpha \beta\left(k_{t+1} y_{t+1}^{n}-k_{t+1} k_{t+1}^{n}\right)+\left[\frac{\alpha \beta}{1-\beta(1-\delta)}\right]\left[\frac{\sigma}{\mathrm{c}}\right] k_{t+1} y_{t+1}^{n}+\left[\frac{\alpha \beta}{1-\beta(1-\delta)}\right]\left[-\frac{\sigma}{\mathrm{c}}\right] k_{t+1} y_{t}^{n} \\
& +\left[\frac{\alpha \beta}{1-\beta(1-\delta)}\right]\left[-\frac{\sigma}{\mathrm{c}} \cdot \frac{1-\mathrm{c}}{\delta}\right] k_{t+1} k_{t+2}^{n}+\left[\frac{\alpha \beta}{1-\beta(1-\delta)}\right]\left[\frac{\sigma}{\mathrm{c}} \cdot \frac{1-\mathrm{c}}{\delta}\right] k_{t+1} k_{t+1}^{n} \\
& \left.\left.+\left[\frac{\alpha \beta}{1-\beta(1-\delta)}\right]\left[\frac{\sigma}{\mathrm{c}} \cdot \frac{1-\mathrm{c}}{\delta}(1-\delta)\right] k_{t+1} k_{t+1}^{n}+\left[\frac{\alpha \beta}{1-\beta(1-\delta)}\right]\left[-\frac{\sigma}{\mathrm{c}} \cdot \frac{1-\mathrm{c}}{\delta}(1-\delta)\right] k_{t+1} k_{t}^{n}\right)\right] \\
& + \text { t.i.p. }+\mathcal{O}\left(\|\xi\|^{3}\right) \text {. }
\end{aligned}
$$

This simplifies to:

$$
\begin{aligned}
\left(\frac{C_{*}^{1-\sigma}}{\mathrm{c}}\right)^{-1} E_{0}\left[\sum_{t=0}^{\infty} \beta^{t}\left(\frac{1}{1-\sigma}\left(C_{t}\right)^{1-\sigma} e^{a \chi_{t}^{c}}-\frac{1}{1+s}\left(H_{t}\right)^{1+s} e^{b \chi_{t}^{h}}\right)\right] \\
=E_{0}\left[\sum _ { t = 0 } ^ { \infty } \beta ^ { t } \left(-\frac{1}{2}\left[\frac{s+\alpha}{1-\alpha}+\frac{\sigma}{\mathrm{c}}\right]\left(y_{t}-y_{t}^{n}\right)^{2}-\frac{1}{2}\left[\frac{\sigma}{\mathrm{c}}\left(\frac{\alpha \beta}{1-\beta(1-\delta)}\right)^{2}+\alpha \beta\right]\left(k_{t+1}-k_{t+1}^{n}\right)^{2}\right.\right. \\
-\frac{1}{2}\left[\frac{\sigma}{\mathrm{c}}\left(\frac{\alpha \beta(1-\delta)}{1-\beta(1-\delta)}\right)^{2}+\frac{\alpha^{2}(1+s)}{1-\alpha}\right]\left(k_{t}-k_{t}^{n}\right)^{2}-\frac{1}{2} \cdot \frac{1}{1-\alpha}\left(\frac{1}{\theta}+\frac{\alpha}{1-\alpha}\right) \operatorname{var}_{z} y_{t}(z)
\end{aligned}
$$




$$
\begin{aligned}
& +\left[-\frac{\sigma}{\mathrm{c}} \cdot \frac{\alpha \beta(1-\delta)}{1-\beta(1-\delta)}+\frac{\alpha(1+s)}{1-\alpha}\right]\left(y_{t}-y_{t}^{n}\right)\left(k_{t}-k_{t}^{n}\right) \\
& +\left[\frac{\sigma}{\mathrm{c}} \cdot \frac{\alpha \beta}{1-\beta(1-\delta)}\right]\left(y_{t}-y_{t}^{n}\right)\left(k_{t+1}-k_{t+1}^{n}\right) \\
& \left.\left.+\left[\frac{\sigma}{\mathrm{c}}\left(\frac{\alpha \beta}{1-\beta(1-\delta)}\right)^{2}(1-\delta)\right]\left(k_{t}-k_{t}^{n}\right)\left(k_{t+1}-k_{t+1}^{n}\right)\right)\right]+ \text { t.i.p. }+\mathcal{O}\left(\|\xi\|^{3}\right) .
\end{aligned}
$$

The complete derivation of this relation is given in appendix A. Equation (54) can also be written in terms of deviations of current investment, output, and the existing capital stock from their respective natural-rate levels; specifically as,

$$
\begin{aligned}
\left(\frac{C_{*}^{1-\sigma}}{\mathrm{c}}\right)^{-1} & E_{0}\left[\sum_{t=0}^{\infty} \beta^{t}\left(\frac{1}{1-\sigma}\left(C_{t}\right)^{1-\sigma} e^{a \chi_{t}^{c}}-\frac{1}{1+s}\left(H_{t}\right)^{1+s} e^{b \chi_{t}^{h}}\right)\right] \\
=E_{0}\left[\sum_{t=0}^{\infty} \beta^{t}(\right. & -\frac{1}{2}\left[\frac{s+\alpha}{1-\alpha}+\frac{\sigma}{\mathrm{c}}\right]\left(y_{t}-y_{t}^{n}\right)^{2}-\frac{1}{2}\left[\frac{\sigma}{\mathrm{c}}\left(\frac{\alpha \beta}{1-\beta(1-\delta)}\right)^{2}+\alpha \beta\right] \delta^{2}\left(i_{t}-i_{t}^{n}\right)^{2} \\
& -\frac{1}{2}\left[\alpha \beta(1-\delta)^{2}+\frac{\alpha^{2}(1+s)}{1-\alpha}\right]\left(k_{t}-k_{t}^{n}\right)^{2}-\frac{1}{2} \cdot \frac{1}{1-\alpha}\left(\frac{1}{\theta}+\frac{\alpha}{1-\alpha}\right) \operatorname{var}_{z} y_{t}(z) \\
+ & {\left[\frac{\alpha(1+s)}{1-\alpha}\right]\left(y_{t}-y_{t}^{n}\right)\left(k_{t}-k_{t}^{n}\right)+\left[\frac{\sigma}{\mathrm{c}} \cdot \frac{\alpha \beta \delta}{1-\beta(1-\delta)}\right]\left(y_{t}-y_{t}^{n}\right)\left(i_{t}-i_{t}^{n}\right) } \\
+ & {\left.\left.[\alpha \beta \delta(1-\delta)]\left(k_{t}-k_{t}^{n}\right)\left(i_{t}-i_{t}^{n}\right)\right)\right]+ \text { t.i.p. }+\mathcal{O}\left(\|\xi\|^{3}\right) . }
\end{aligned}
$$

It is worth noting from equations (54) and (55) that when the definition of the natural-rate variables follows that of Neiss and Nelson our expressions for the second-order approximation to the utility function can (apart from the output dispersion term) be completely characterized in terms of gaps between variables' actual and natural-rate values.

\subsubsection{Derivation Using the Woodford Natural Rate}

Under Woodford's definition of the natural rate, we obtain the following second-order approximation to the overall utility function:

$$
\begin{gathered}
\left(\frac{C_{*}^{1-\sigma}}{\mathrm{c}}\right)^{-1} E_{0}\left[\sum_{t=0}^{\infty} \beta^{t}\left(\frac{1}{1-\sigma}\left(C_{t}\right)^{1-\sigma} e^{a \chi_{t}^{c}}-\frac{1}{1+s}\left(H_{t}\right)^{1+s} e^{b \chi_{t}^{h}}\right)\right] \\
=E_{0}\left[\sum _ { t = 0 } ^ { \infty } \beta ^ { t } \left(\frac{1}{2}\left(1-\frac{\sigma}{\mathrm{c}}\right) y_{t}^{2}+\frac{1}{2}\left(-\frac{1-\mathrm{c}}{\delta}-\frac{\sigma}{\mathrm{c}}\left(\frac{1-\mathrm{c}}{\delta}\right)^{2}\right) k_{t+1}^{2}+\frac{1}{2}\left(\frac{1-\mathrm{c}}{\delta}(1-\delta)-\frac{\sigma}{\mathrm{c}}\left(\frac{1-\mathrm{c}}{\delta}\right)^{2}(1-\delta)^{2}\right) k_{t}^{2}\right.\right. \\
-\frac{\sigma}{\mathrm{c}} \cdot \frac{1-\mathrm{c}}{\delta}(1-\delta) y_{t} k_{t}+\frac{\sigma}{\mathrm{c}} \cdot \frac{1-\mathrm{c}}{\delta} y_{t} k_{t+1}+\frac{\sigma}{\mathrm{c}}\left(\frac{1-\mathrm{c}}{\delta}\right)^{2}(1-\delta) k_{t} k_{t+1}+\frac{1}{2} \cdot \frac{-\alpha}{1-\alpha} \alpha(1+s) k_{t}^{2} \\
+\frac{1}{2} \cdot \frac{-(1+s)}{1-\alpha} y_{t}^{2}+\frac{\alpha(1+s)}{1-\alpha} k_{t} y_{t}-\frac{1}{2} \cdot \frac{1}{1-\alpha}\left(\frac{1}{\theta}+\frac{\alpha}{1-\alpha}\right) \operatorname{var}_{z} y_{t}(z)
\end{gathered}
$$




$$
\begin{aligned}
& +\left[\frac{s+\alpha}{1-\alpha}+\frac{\sigma}{\mathrm{c}}\right] y_{t} y_{t}^{n}+\left[-\frac{\alpha(1+s)}{1-\alpha}+\frac{\sigma}{\mathrm{c}} \cdot \frac{1-\mathrm{c}}{\delta}(1-\delta)\right] y_{t} k_{t}+\left[-\frac{\sigma}{\mathrm{c}} \cdot \frac{1-\mathrm{c}}{\delta}\right] y_{t} k_{t+1}^{n} \\
& +\left[\frac{\beta \alpha(1-\delta)}{1-\beta(1-\delta)}\right]\left[\frac{s+\alpha}{1-\alpha}+\frac{\sigma}{\mathrm{c}}\right] k_{t} y_{t}^{n}+\left[\frac{\beta \alpha(1-\delta)}{1-\beta(1-\delta)}\right]\left[-\frac{\alpha(1+s)}{1-\alpha}+\frac{\sigma}{\mathrm{c}} \cdot \frac{1-\mathrm{c}}{\delta}(1-\delta)\right] k_{t}^{2} \\
& +\left[\frac{\beta \alpha(1-\delta)}{1-\beta(1-\delta)}\right]\left[-\frac{\sigma}{\mathrm{c}} \cdot \frac{1-\mathrm{c}}{\delta}\right] k_{t} k_{t+1}^{n}+\left[\frac{\alpha}{1-\beta(1-\delta)}\right]\left[-\frac{s+\alpha}{1-\alpha}-\frac{\sigma}{\mathrm{c}}\right] k_{t} y_{t}^{n} \\
& +\left[\frac{\alpha}{1-\beta(1-\delta)}\right]\left[\frac{\alpha(1+s)}{1-\alpha}-\frac{\sigma}{\mathrm{c}} \cdot \frac{1-\mathrm{c}}{\delta}(1-\delta)\right] k_{t}^{2}+\left[\frac{\alpha}{1-\beta(1-\delta)}\right]\left[\frac{\sigma}{\mathrm{c}} \cdot \frac{1-\mathrm{c}}{\delta}\right] k_{t} k_{t+1}^{n} \\
& -\alpha \beta\left(k_{t+1} y_{t+1}^{n}-k_{t+1} k_{t+1}^{n}\right)+\left[\frac{\alpha \beta}{1-\beta(1-\delta)}\right]\left[\frac{\sigma}{\mathrm{c}}\right] k_{t+1} y_{t+1}^{n}+\left[\frac{\alpha \beta}{1-\beta(1-\delta)}\right]\left[-\frac{\sigma}{\mathrm{c}}\right] k_{t+1} y_{t}^{n} \\
& +\left[\frac{\alpha \beta}{1-\beta(1-\delta)}\right]\left[-\frac{\sigma}{\mathrm{c}} \cdot \frac{1-\mathrm{c}}{\delta}\right] k_{t+1} k_{t+2}^{n}+\left[\frac{\alpha \beta}{1-\beta(1-\delta)}\right]\left[\frac{\sigma}{\mathrm{c}} \cdot \frac{1-\mathrm{c}}{\delta}\right] k_{t+1} k_{t+1}^{n} \\
& \left.\left.+\left[\frac{\alpha \beta}{1-\beta(1-\delta)}\right]\left[\frac{\sigma}{\mathrm{c}} \cdot \frac{1-\mathrm{c}}{\delta}(1-\delta)\right] k_{t+1}^{2}+\left[\frac{\alpha \beta}{1-\beta(1-\delta)}\right]\left[-\frac{\sigma}{\mathrm{c}} \cdot \frac{1-\mathrm{c}}{\delta}(1-\delta)\right] k_{t+1} k_{t}\right)\right] \\
& + \text { t.i.p. }+\mathcal{O}\left(\|\xi\|^{3}\right) .
\end{aligned}
$$

As shown in appendix A, this simplifies to:

$$
\begin{aligned}
\left(\frac{C_{*}^{1-\sigma}}{\mathrm{c}}\right)^{-1} & E_{0}\left[\sum_{t=0}^{\infty} \beta^{t}\left(\frac{1}{1-\sigma}\left(C_{t}\right)^{1-\sigma} e^{a \chi_{t}^{c}}-\frac{1}{1+s}\left(H_{t}\right)^{1+s} e^{b \chi_{t}^{h}}\right)\right] \\
=E_{0}\left[\sum_{t=0}^{\infty} \beta^{t}\right. & \left(-\frac{1}{2}\left[\frac{s+\alpha}{1-\alpha}+\frac{\sigma}{\mathrm{c}}\right]\left(y_{t}-y_{t}^{n}\right)^{2}-\frac{1}{2}\left[\frac{\sigma}{\mathrm{c}}\left(\frac{\alpha \beta}{1-\beta(1-\delta)}\right)^{2}+\alpha \beta\right]\left(k_{t+1}-k_{t+1}^{n}\right)^{2}\right. \\
+ & \frac{1}{2}\left[\frac{\sigma}{\mathrm{c}}\left(\frac{\alpha \beta(1-\delta)}{1-\beta(1-\delta)}\right)^{2}+\frac{\alpha^{2}(1+s)}{1-\alpha}\right] k_{t}^{2}-\frac{1}{2} \cdot \frac{1}{1-\alpha}\left(\frac{1}{\theta}+\frac{\alpha}{1-\alpha}\right) \operatorname{var}_{z} y_{t}(z) \\
+ & {\left[\frac{\sigma}{\mathrm{c}} \cdot \frac{\alpha \beta(1-\delta)}{1-\beta(1-\delta)}-\frac{\alpha(1+s)}{1-\alpha}\right] k_{t} y_{t}^{n}-\left[\frac{\sigma}{\mathrm{c}}\left(\frac{\alpha \beta}{1-\beta(1-\delta)}\right)^{2}(1-\delta)\right] k_{t} k_{t+1}^{n} } \\
+ & {\left.\left[\frac{\sigma}{\mathrm{c}} \cdot \frac{\alpha \beta}{1-\beta(1-\delta)}\right]\left(y_{t}-y_{t}^{n}\right)\left(k_{t+1}-k_{t+1}^{n}\right)\right]+ \text { t.i.p. }+\mathcal{O}\left(\|\xi\|^{3}\right), }
\end{aligned}
$$

or, alternatively:

$$
\begin{aligned}
\left(\frac{C_{*}^{1-\sigma}}{\mathrm{c}}\right)^{-1} & E_{0}\left[\sum_{t=0}^{\infty} \beta^{t}\left(\frac{1}{1-\sigma}\left(C_{t}\right)^{1-\sigma} e^{a \chi_{t}^{c}}-\frac{1}{1+s}\left(H_{t}\right)^{1+s} e^{b \chi_{t}^{h}}\right)\right] \\
=E_{0}\left[\sum_{t=0}^{\infty} \beta^{t}\right. & \left(-\frac{1}{2}\left[\frac{s+\alpha}{1-\alpha}+\frac{\sigma}{\mathrm{c}}\right]\left(y_{t}-y_{t}^{n}\right)^{2}-\frac{1}{2}\left[\frac{\sigma}{\mathrm{c}}\left(\frac{\alpha \beta \delta}{1-\beta(1-\delta)}\right)^{2}+\alpha \beta \delta^{2}\right]\left(i_{t}-i_{t}^{n}\right)^{2}\right. \\
& -\frac{1}{2}\left[\frac{\sigma}{\mathrm{c}}\left(\frac{\alpha \beta(1-\delta)}{1-\beta(1-\delta)}\right)^{2}+\frac{\alpha^{2}(1+s)}{1-\alpha}\right] k_{t}^{2}-\frac{1}{2} \cdot \frac{1}{1-\alpha}\left(\frac{1}{\theta}+\frac{\alpha}{1-\alpha}\right) \operatorname{var}_{z} y_{t}(z) \\
+ & {\left.\left.\left[\frac{\sigma}{\mathrm{c}} \cdot \frac{\alpha \beta \delta}{1-\beta(1-\delta)}\right]\left(y_{t}-y_{t}^{n}\right)\left(i_{t}-i_{t}^{n}\right)+\left[\sigma \frac{\alpha \beta(1-\delta)}{1-\beta(1-\delta)}\right] k_{t} c_{t}^{n}-[\alpha(1+s)] k_{t} h_{t}^{n}\right)\right] }
\end{aligned}
$$




$$
+ \text { t.i.p. }+\mathcal{O}\left(\|\xi\|^{3}\right)
$$

In contrast to the previous case, when the definition of natural-rate variables follows Woodford (2003), our expressions for the second-order approximation to the utility function cannot be entirely characterized with gap terms (along with an output dispersion term): In addition to these gaps, the squared actual capital stock and various actual and natural-rate cross products enter the welfare expression. Intuitively, the actual state of the economy has a separate influence on welfare in this case.

\section{The Welfare Criterion in a Sticky-Price Model}

Up to this point, we have not discussed what role sticky prices would play in our derivation of the loss function. Sticky prices imply that there will be a relationship between inflation and the output dispersion term $\operatorname{var}_{z} y_{t}(z)$ in our loss function expressions in section 5.3. In addition, it is of independent interest to determine how the gap terms in the loss functions that we have derived are related to the gap terms that determine inflation in the Phillips curve.

As we noted in section 2.2, the only part of the model that changes when sticky prices are introduced is the intermediate-good producing firm's profit-maximization problem, which must now reflect the fact that prices are fixed in advance. To model sticky prices, we assume Calvo (1983) pricing (as modified by Yun, 1996), in which in any period a randomly selected fraction $1-\gamma$ of firms can reset their price, while the remaining fraction $\gamma$ are constrained to charge their existing price (which is indexed to the steady-state inflation rate). In this setup, a firm that is able to reset its price in period $t$ takes as given its marginal cost $M C_{t}(z)$, the aggregate price level $P_{t}$, and aggregate output $Y_{t}$, and solves:

$$
\max _{\left\{P_{t}(z)\right\}} \sum_{k=0}^{\infty} \gamma^{k} E_{t}\left[Q_{0, k}\left(\left(P_{t}(z)-(1-\tau) M C_{t}(z)\right) Y_{t}(z)\right)\right] \text { s.t. } Y_{t}(z)=Y_{t}\left(\frac{P_{t}(z)}{P_{t}}\right)^{-\theta},
$$

where $Q_{t, t+v}$ denotes the nominal stochastic discount factor (which firms also take as given). ${ }^{5}$

Equilibrium in the sticky-price model is an allocation $\left\{C_{t}, I_{t}, Y_{t}, H_{t},\left\{H_{t}(z)\right\}_{z=0}^{1}, K_{t},\left\{K_{t}(z)\right\}_{z=0}^{1}\right\}_{t=0}^{\infty}$ and a sequence of values $\left\{\Pi_{t}, W_{t} / P_{t}, R_{t}^{k} / P_{t},\left\{M C_{t}(z)\right\}_{z=0}^{1}, R_{t}\right\}_{t=0}^{\infty}$ that satisfy the following conditions: (i) the final-good producing firm solves (5); (ii) all intermediate-good producing

\footnotetext{
${ }^{5}$ If we write the marginal utility of consumption as $M U_{t}$, then the nominal stochastic discount factor $Q_{t, t+v}$ equals $\frac{\beta^{v} M U_{t+v} / P_{t+v}}{M U_{t} / P_{t}}$.
} 
firms solve (7) and (59); (iii) the household solves (10); (iv) the monetary authority sets the nominal interest rate in a manner that ensures a determinate equilibrium; (v) the goods market clears; and, (vi) all factor markets clear. Agents take as given the inital value of $K_{0}$, and the sequence of shocks $\left\{X_{t}, \chi_{t}^{c}, \chi_{t}^{h}\right\}_{t=0}^{\infty}$ that hit the economy.

The equilibrium conditions in the sticky-price economy are as follows. The first-order condition from the final-good producing firm's cost-minimization problem is given by equation (6). The first-order conditions from the the intermediate-good producing firm's costminimization problem are given by equations (11), (12), and (13), while the first-order condition from its profit-maximization problem is given by:

$$
P_{t}(z)=\frac{\sum_{k=0}^{\infty} \gamma E_{t}\left[Q_{0, t} M C_{t+k}(z) \theta Y_{t+k}\right]}{\sum_{k=0}^{\infty} \gamma E_{t}\left[Q_{0, t}(\theta-1) Y_{t+k}\right]} .
$$

In addition, the first-order conditions from the household's utility-maximization problem are given by equations (3), (16), (17), and (18). Market clearing in the goods market is given by equation (19), while in factor markets it is given by equation (20). The steady-state conditions for the sticky-price model are identical to those given in section 2.4.

The log-linearized first-order conditions from the intermediate-good producing firm's costminimization problem are still given by equations (23), (24), and (25). The first-order condition from the intermediate-good producing firm's profit-maximization problem (equation 60) log-linearizes to:

$$
\pi_{t}=\beta E_{t} \pi_{t+1}+\frac{(1-\gamma)(1-\gamma \beta)}{\gamma} m c_{t}+\mathcal{O}\left(\|\xi\|^{2}\right), \quad \text { where } m c_{t}=\alpha r_{t}^{k}+(1-\alpha) w_{t}+x_{t}+\mathcal{O}\left(\|\xi\|^{2}\right)
$$

The log-linearized first-order conditions from the household's utility-maximization problem are still given by equations (27), (28), (29), and (30), while goods market clearing is still given by (31).

\subsection{The Phillips Curve in Terms of "Gap" Variables}

We now wish to compare the gap terms that enter the approximate loss functions that we have derived with the gap terms that enter the Phillips curve. From the expression for marginal cost given in equation (61) as well as from equations (23), (25), (28), (30), and (31), we can write:

$m c_{t}=\left[\frac{s+\alpha}{1-\alpha}+\frac{\sigma}{\mathrm{c}}\right] y_{t}-\left[\frac{\alpha(1+s)}{1-\alpha}-\frac{\sigma}{\mathrm{c}} \cdot \frac{1-\mathrm{c}}{\delta}(1-\delta)\right] k_{t}-\left[\frac{\sigma}{\mathrm{c}} \cdot \frac{1-\mathrm{c}}{\delta}\right] k_{t+1}-a \chi_{t}^{c}+b \chi_{t}^{h}-\frac{1+s}{1-\alpha} x_{t}+\mathcal{O}\left(\|\xi\|^{2}\right)$. 
The flexible-price equivalent to equation (62) using the Neiss-Nelson definition of the natural rate is:

$$
\begin{aligned}
m c_{t}^{n} & =\left[\frac{s+\alpha}{1-\alpha}+\frac{\sigma}{\mathrm{c}}\right] y_{t}^{n}-\left[\frac{\alpha(1+s)}{1-\alpha}-\frac{\sigma}{\mathrm{c}} \cdot \frac{1-\mathrm{c}}{\delta}(1-\delta)\right] k_{t}^{n}-\left[\frac{\sigma}{\mathrm{c}} \cdot \frac{1-\mathrm{c}}{\delta}\right] k_{t+1}^{n}-a \chi_{t}^{c}+b \chi_{t}^{h}-\frac{1+s}{1-\alpha} x_{t}+\mathcal{O}\left(\|\xi\|^{2}\right) \\
& =0+\mathcal{O}\left(\|\xi\|^{2}\right),
\end{aligned}
$$

while using Woodford's definition it is:

$$
\begin{aligned}
m c_{t}^{n} & =\left[\frac{s+\alpha}{1-\alpha}+\frac{\sigma}{\mathrm{c}}\right] y_{t}^{n}-\left[\frac{\alpha(1+s)}{1-\alpha}-\frac{\sigma}{\mathrm{c}} \cdot \frac{1-\mathrm{c}}{\delta}(1-\delta)\right] k_{t}-\left[\frac{\sigma}{\mathrm{c}} \cdot \frac{1-\mathrm{c}}{\delta}\right] k_{t+1}^{n}-a \chi_{t}^{c}+b \chi_{t}^{h}-\frac{1+s}{1-\alpha} x_{t}+\mathcal{O}\left(\|\xi\|^{2}\right) \\
& =0+\mathcal{O}\left(\|\xi\|^{2}\right) .
\end{aligned}
$$

Note that in both (63) and (64), $m c_{t}^{n}=0$ - we are in the flexible-price model-which makes these two equations identical to (34) and (36).

Since $m c_{t}^{n}=0$, we can add it to our Phillips curve expression (equation 61) without its altering anything. Hence,

$$
\pi_{t}=\beta E_{t} \pi_{t+1}+\frac{(1-\gamma)(1-\gamma \beta)}{\gamma} \cdot\left(m c_{t}-m c_{t}^{n}\right)+\mathcal{O}\left(\|\xi\|^{2}\right)
$$

This implies that when we use the Neiss-Nelson definition of the natural rate of output, our Phillips curve in terms of gap terms is:

$$
\begin{array}{r}
\pi_{t}=\beta E_{t} \pi_{t+1}+\frac{(1-\gamma)(1-\gamma \beta)}{\gamma}\left(\left[\frac{s+\alpha}{1-\alpha}+\frac{\sigma}{\mathrm{c}}\right]\left(y_{t}-y_{t}^{n}\right)-\left[\frac{\alpha(1+s)}{1-\alpha}-\frac{\sigma}{\mathrm{c}} \cdot \frac{1-\mathrm{c}}{\delta}(1-\delta)\right]\left(k_{t}-k_{t}^{n}\right)\right. \\
\left.-\left[\frac{\sigma}{\mathrm{c}} \cdot \frac{1-\mathrm{c}}{\delta}\right]\left(k_{t+1}-k_{t+1}^{n}\right)\right)+\mathcal{O}\left(\|\xi\|^{2}\right),(66)
\end{array}
$$

or

$$
\begin{aligned}
\pi_{t}=\beta E_{t} \pi_{t+1}+\frac{(1-\gamma)(1-\gamma \beta)}{\gamma}( & {\left[\frac{s+\alpha}{1-\alpha}+\frac{\sigma}{\mathrm{c}}\right]\left(y_{t}-y_{t}^{n}\right) } \\
& \left.-\left[\frac{\alpha(1+s)}{1-\alpha}\right]\left(k_{t}-k_{t}^{n}\right)-\left[\frac{\sigma}{\mathrm{c}}(1-\mathrm{c})\right]\left(i_{t}-i_{t}^{n}\right)\right)+\mathcal{O}\left(\|\xi\|^{2}\right),(67
\end{aligned}
$$

where the latter expression is written in terms of the "investment gap." When we use Woodford's definition, our Phillips curve is:

$$
\pi_{t}=\beta E_{t} \pi_{t+1}+\frac{(1-\gamma)(1-\gamma \beta)}{\gamma}\left(\left[\frac{s+\alpha}{1-\alpha}+\frac{\sigma}{\mathrm{c}}\right]\left(y_{t}-y_{t}^{n}\right)-\left[\frac{\sigma}{\mathrm{c}} \cdot \frac{1-\mathrm{c}}{\delta}\right]\left(k_{t+1}-k_{t+1}^{n}\right)\right)+\mathcal{O}\left(\|\xi\|^{2}\right),
$$

or

$$
\pi_{t}=\beta E_{t} \pi_{t+1}+\frac{(1-\gamma)(1-\gamma \beta)}{\gamma}\left(\left[\frac{s+\alpha}{1-\alpha}+\frac{\sigma}{\mathrm{c}}\right]\left(y_{t}-y_{t}^{n}\right)-\left[\frac{\sigma}{\mathrm{c}}(1-\mathrm{c})\right]\left(i_{t}-i_{t}^{n}\right)\right)+\mathcal{O}\left(\|\xi\|^{2}\right) .
$$


We see, therefore, that the same gap terms enter both the loss function and the Phillips curve when we use the Neiss-Nelson definition of the natural rate of output. When we use Woodford's definition, the gaps that determine inflation also appear in the loss function; however, additional terms are present there as well. ${ }^{6}$

\subsection{Output Dispersion and Inflation}

We now briefly consider the relationship between inflation and the output dispersion term $\operatorname{var}_{z} y_{t}(z)=\operatorname{var}_{z} \ln Y_{t}(z)$ that appears in the equations of section 5.3.

It turns out that the relationship between output dispersion and inflation is unaltered by the presence of capital; as a result, the analysis is identical to that outlined in Chapter 6 of Woodford (2003). In particular, the demand for each intermediate good (equation 6) implies that $\operatorname{var}_{z} \ln Y_{t}(z)=\theta^{2} \operatorname{var}_{z} \ln P_{t}(z)$, while the recursive nature of the price level under Calvo contracting implies that

$$
\operatorname{var}_{z} \ln P_{t}(z)=\left(\frac{\gamma}{1-\gamma}\right) \sum_{s=0}^{t} \gamma^{t-s} \pi_{s}^{2}+\text { t.i.p }+\mathcal{O}\left(\|\xi\|^{3}\right)
$$

so that

$$
\frac{1}{1-\alpha}\left(\frac{1}{\theta}+\frac{\alpha}{1-\alpha}\right) \operatorname{var}_{z} y_{t}(z)=\frac{\theta}{1-\alpha}\left(1+\frac{\theta \alpha}{1-\alpha}\right)\left(\frac{\gamma}{1-\gamma}\right) \sum_{s=0}^{t} \gamma^{t-s} \pi_{s}^{2}+\text { t.i.p }+\mathcal{O}\left(\|\xi\|^{3}\right),
$$

or

$$
\begin{aligned}
& E_{t}\left[\sum_{t=0}^{\infty} \beta^{t}\right.\left.\frac{1}{1-\alpha}\left(\frac{1}{\theta}+\frac{\alpha}{1-\alpha}\right) \operatorname{var}_{z} y_{t}(z)\right] \\
& \quad=E_{t}\left[\sum_{t=0}^{\infty} \beta^{t} \frac{\theta}{1-\alpha}\left(1+\frac{\theta \alpha}{1-\alpha}\right)\left(\frac{\gamma}{(1-\gamma)(1-\beta \gamma)}\right) \pi_{t}^{2}\right]+\text { t.i.p }+\mathcal{O}\left(\|\xi\|^{3}\right) \\
& \quad=\left(\frac{1}{1-\beta}\right)\left(\frac{\theta}{1-\alpha}\right)\left(1+\frac{\theta \alpha}{1-\alpha}\right)\left(\frac{\gamma}{(1-\gamma)(1-\beta \gamma)}\right) \operatorname{var}\left(\pi_{t}\right)+\text { t.i.p }+\mathcal{O}\left(\|\xi\|^{3}\right)
\end{aligned}
$$

which we can use to replace the $\operatorname{var}_{z} y_{t}(z)$ terms in the equations in section 5.3.

\footnotetext{
${ }^{6}$ Hence, care must be taken in extrapolating Woodford's (2003) point that one can associate the output gap in the new-Keynesian Phillips curve with the gap that belongs in a Taylor-style interest rate feedback rule. As the preceding analysis indicates, such a conclusion only extends to a model with endogenous capital in a very loose sense (because cross products of the gap terms now appear in the loss function) and, even in this more limited sense, only obtains for a specific definition of potential output.
} 


\subsection{The Utility-based Welfare Criterion}

Taking expectations of equation (55) implies that

$$
\begin{aligned}
(1-\beta) & \left(\frac{C_{*}^{1-\sigma}}{\mathrm{c}}\right)^{-1} E_{0}\left[\sum_{t=0}^{\infty} \beta^{t}\left(\frac{1}{1-\sigma}\left(C_{t}\right)^{1-\sigma} e^{a \chi_{t}^{c}}-\frac{1}{1+s}\left(H_{t}\right)^{1+s} e^{b \chi_{t}^{h}}\right)\right] \\
= & -\frac{1}{2}\left(\frac{s+\alpha}{1-\alpha}+\frac{\sigma}{\mathrm{c}}\right) \operatorname{var}\left(y_{t}-y_{t}^{n}\right)-\frac{1}{2} \cdot \frac{\sigma}{\mathrm{c}}\left(\frac{\alpha \beta \delta}{1-\beta(1-\delta)}\right)^{2} \operatorname{var}\left(i_{t}-i_{t}^{n}\right) \\
& -\frac{1}{2} \cdot \frac{\theta}{1-\alpha}\left(1+\frac{\theta \alpha}{1-\alpha}\right) \frac{\gamma}{(1-\gamma)(1-\beta \gamma)} \operatorname{var}\left(\pi_{t}\right)+\frac{\sigma}{\mathrm{c}} \cdot \frac{\alpha \beta \delta}{1-\beta(1-\delta)} \operatorname{cov}\left(y_{t}-y_{t}^{n}, i_{t}-i_{t}^{n}\right) \\
& -\frac{1}{2} \cdot \frac{\alpha \beta \delta^{2}}{1-\beta(1-\delta)^{2}} \cdot \frac{1+\alpha s}{1-\alpha} \sum_{j=-\infty}^{\infty}(\beta(1-\delta))^{j} \operatorname{cov}\left(i_{t}-i_{t}^{n}, i_{t-j}-i_{t-j}^{n}\right) \\
& +\frac{\delta}{1-\delta} \alpha \frac{\alpha(1+s)}{1-\alpha} \sum_{j=1}^{\infty}(\beta(1-\delta))^{j} \operatorname{cov}\left(i_{t-j}-i_{t-j}^{n}, y_{t}-y_{t}^{n}\right)+\text { t.i.p. }+\mathcal{O}\left(\|\xi\|^{3}\right),
\end{aligned}
$$

where the natural-rate variables follow the Neiss-Nelson natural rate definition. Alternatively, under the Woodford natural rate definition, we have that

$$
\begin{aligned}
(1-\beta) & \left(\frac{C_{*}^{1-\sigma}}{\mathrm{c}}\right)^{-1} E_{0}\left[\sum_{t=0}^{\infty} \beta^{t}\left(\frac{1}{1-\sigma}\left(C_{t}\right)^{1-\sigma} e^{a \chi_{t}^{c}}-\frac{1}{1+s}\left(H_{t}\right)^{1+s} e^{b \chi_{t}^{h}}\right)\right] \\
= & -\frac{1}{2}\left(\frac{s+\alpha}{1-\alpha}+\frac{\sigma}{\mathrm{c}}\right) \operatorname{var}\left(y_{t}-y_{t}^{n}\right)-\frac{1}{2}\left(\frac{\sigma}{\mathrm{c}}\left(\frac{\alpha \beta \delta}{1-\beta(1-\delta)}\right)^{2}+\alpha \beta \delta^{2}\right) \operatorname{var}\left(i_{t}-i_{t}^{n}\right) \\
& -\frac{1}{2} \cdot \frac{\theta}{1-\alpha}\left(1+\frac{\theta \alpha}{1-\alpha}\right) \frac{\gamma}{(1-\gamma)(1-\beta \gamma)} \operatorname{var}\left(\pi_{t}\right)+\frac{\sigma}{\mathrm{c}} \cdot \frac{\alpha \beta \delta}{1-\beta(1-\delta)} \operatorname{cov}\left(y_{t}-y_{t}^{n}, i_{t}-i_{t}^{n}\right) \\
& -\frac{1}{2}\left(\frac{\sigma}{\mathrm{c}}\left(\frac{\alpha \beta(1-\delta)}{1-\beta(1-\delta)}\right)^{2}+\frac{\alpha^{2}(1+s)}{1-\alpha}\right) \operatorname{var}\left(k_{t}\right) \\
+ & \sigma \frac{\alpha \beta(1-\delta)}{1-\beta(1-\delta)} \operatorname{cov}\left(k_{t}, c_{t}^{n}\right)-\alpha(1+s) \operatorname{cov}\left(k_{t}, h_{t}^{n}\right)+\text { t.i.p. }+\mathcal{O}\left(\|\xi\|^{3}\right)
\end{aligned}
$$

(which follows from taking expectations of equation 58).

For both definitions of the natural rate variables, the variance of the output gap - written as $\operatorname{var}\left(y_{t}-y_{t}^{n}\right)$ - and the variance of the inflation rate - $\operatorname{var}\left(\pi_{t}\right)$ - have a detrimental effect on household utility, just as they do in the model without endogenous capital accumulation. In addition, for both natural rate definitions, the variance of the investment spending gap$\operatorname{var}\left(i_{t}-i_{t}^{n}\right)$-also reduces household utility, implying that the composition of output-not merely its aggregate value - has welfare implications in the model with endogenous capital. (Of course, this reflects the fact that current investment-and, hence, next period's capital 
stock - directly affects overall utility.) In addition, the comovement between the contemporaneous values of the investment and output gaps also affects household utility, this time positively. Because consumption affects utility, it can be preferable for a given output gap to spill over into the investment gap rather than into the consumption gap. The weight on this term depends on the intertemporal elasticity of substitution $\sigma$ as well as the steady-state ratio of investment to consumption spending $\frac{1-c}{c}$ (recall equation 22).

When the natural rate terms are characterized according to the Neiss-Nelson definition, the covariance of investment gaps across time also has a detrimental impact on utility. This is because under the Neiss-Nelson natural-rate definition (in which the natural rate is defined with reference to the flexible-price model's capital stock) positive covariances between investment gaps imply that the actual capital stock - and hence all model variables - is moving progressively further away from its welfare-maximizing natural rate level. By contrast, a positive covariance between the output gap and previous investment gaps affects household utility in a favorable way. Previous levels of investment spending that exceed their natural rate imply a level of the capital stock that is also in excess of its natural rate. If capital covaries with output in this manner, then smaller fluctuations in hours will result, which is itself welfare enhancing. The weight on this term depends on the elasticity of labor sup-

ply $s$, the relative importance of capital and labor in the production process $\frac{\alpha}{1-\alpha}$, and the depreciation rate of previously installed capital $\delta$.

Finally, when natural rate variables are characterized according to Woodford's definition, the variance of deviations of the actual capital stock from its steady-state level reduces utility, as does a positive covariance between capital and the natural rate of hours. In contrast, a positive covariance between capital and the natural rate of consumption is favorable to utility. It is difficult to place an intuitive interpretation on these terms.

\section{Conclusions}

This paper has extended the utility-based welfare criterion developed by Rotemberg and Woodford (1997) and Woodford (2003) to a model with endogenous capital accumulation. By deriving an explicit analytical solution, it has been possible to compare the welfare expression that results in this model to the welfare criteria that are obtained in models where a capital accumulation decision is absent. In addition, the explicit expressions derived here permit us to see to what extent welfare can be characterized in terms of suitably defined gaps between variables' actual and natural rate values. 
As has been shown, the welfare criterion obtained for this model shares several features with the corresponding expressions that have been derived in simpler models. In particular, a criterion can be specified such that welfare losses depend solely on quadratic functions of the model's variables, thus confirming that policy should be oriented toward stabilization of macroeconomic aggregates, rather than toward attaining particular levels of those aggregates. That said, an important difference that obtains in this case is that the composition of output directly affects welfare in the endogenous-capital model - a result that is not present in standard treatments.

Of course, the degree to which these additional considerations will affect the characterization of optimal monetary policy remains an open question. A natural next step, thenwhich is left for future research - is to use the welfare criterion developed here in order to assess optimal policy in the context of more richly specified (and empirically valid) structural macromodels.

\section{References}

[1] Calvo, Guillerno A. (1983). "Staggered Prices in a Utility-Maximizing Framework," Journal of Monetary Economics 12, 383-389.

[2] Neiss, Katherine S. and Edward Nelson (2003). "The Real Interest Rate Gap as an Inflation Indicator," Macroeconomic Dynamics 7, 239-262.

[3] Rotemberg, Julio J. and Michael Woodford (1997). "An Optimization-Based Econometric Framework for the Evaluation of Monetary Policy," NBER Macroeconomics Annual 1997, edited by Ben S. Bernanke and Julio J. Rotemberg. Cambridge, MA: MIT Press.

[4] Woodford, Michael (2001). "The Taylor Rule and Optimal Monetary Policy," American Economic Review 91, 232-237.

[5] Woodford, Michael (2003). Interest and Prices. Princeton, NJ: Princeton University Press.

[6] Yun, Tak (1996). "Nominal Price Rigidity, Money Supply Endogeneity, and Business Cycles," Journal of Monetary Economics 37, 345-370. 


\section{A Appendix}

This appendix provides detailed derivations for several equations in the text.

\section{A.1 Detailing the Steps between Equations (53) and (54)}

It is useful to label all terms in (53). I use the code that second-order model variable terms that are in the loss function via consumption are labeled with a $C$; second-order model variable terms that are in the loss function via hours are labeled with an $H$; and secondorder model variable terms that are in the loss function via the second-order cross-product terms are labeled with an $X$.

$$
\begin{aligned}
& \left(\frac{C_{*}^{1-\sigma}}{\mathrm{c}}\right)^{-1} E_{0}\left[\sum_{t=0}^{\infty} \beta^{t}\left(\frac{1}{1-\sigma}\left(C_{t}\right)^{1-\sigma} e^{a \chi_{t}^{c}}-\frac{1}{1+s}\left(H_{t}\right)^{1+s} e^{b \chi_{t}^{h}}\right)\right]
\end{aligned}
$$

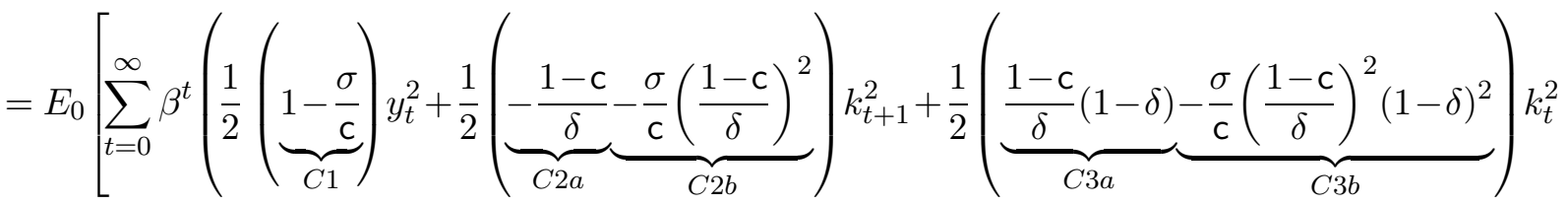

$$
\begin{aligned}
& \underbrace{-\frac{\sigma}{\mathrm{c}} \cdot \frac{1-\mathrm{c}}{\delta}(1-\delta)}_{C 4} y_{t} k_{t}+\underbrace{\frac{\sigma}{\mathrm{c}} \cdot \frac{1-\mathrm{c}}{\delta}}_{C 5} y_{t} k_{t+1}+\underbrace{\frac{\sigma}{\mathrm{c}\left(\frac{1-\mathrm{c}}{\delta}\right)^{2}(1-\delta)}}_{C 6} k_{t} k_{t+1}+\frac{1}{2} \cdot \underbrace{\frac{-\alpha}{1-\alpha} \alpha(1+s)}_{H 1} k_{t}^{2} \\
& +\frac{1}{2} \cdot \underbrace{\frac{-(1+s)}{1-\alpha}}_{H 2} y_{t}^{2}+\underbrace{\frac{\alpha(1+s)}{1-\alpha}}_{H 3} k_{t} y_{t}-\frac{1}{2} \cdot \frac{1}{1-\alpha}\left(\frac{1}{\theta}+\frac{\alpha}{1-\alpha}\right) \operatorname{var}_{z} y_{t}(z) \\
& +\underbrace{\left[\frac{s+\alpha}{1-\alpha}+\frac{\sigma}{\mathrm{c}}\right]}_{X 1} y_{t} y_{t}^{n}+\underbrace{\left[-\frac{\alpha(1+s)}{1-\alpha}+\frac{\sigma}{\mathrm{c}} \cdot \frac{1-\mathrm{c}}{\delta}(1-\delta)\right]}_{X 2} y_{t} k_{t}^{n}+\underbrace{\left[-\frac{\sigma}{\mathrm{c}} \cdot \frac{1-\mathrm{c}}{\delta}\right]}_{X 3} y_{t} k_{t+1}^{n} \\
& +\underbrace{\left[\frac{\beta \alpha(1-\delta)}{1-\beta(1-\delta)}\right]\left[\frac{s+\alpha}{1-\alpha}+\frac{\sigma}{\mathrm{c}}\right]}_{X 4} k_{t} y_{t}^{n}+\underbrace{\left[\frac{\beta \alpha(1-\delta)}{1-\beta(1-\delta)}\right]\left[-\frac{\alpha(1+s)}{1-\alpha}+\frac{\sigma}{\mathrm{c}} \cdot \frac{1-\mathrm{c}}{\delta}(1-\delta)\right]}_{X 5} k_{t} k_{t}^{n} \\
& +\underbrace{\left[\frac{\beta \alpha(1-\delta)}{1-\beta(1-\delta)}\right]\left[-\frac{\sigma}{\mathrm{c}} \cdot \frac{1-\mathrm{c}}{\delta}\right]}_{X 6} k_{t} k_{t+1}^{n}+\underbrace{\left[\frac{\alpha}{1-\beta(1-\delta)}\right]\left[-\frac{s+\alpha}{1-\alpha}-\frac{\sigma}{\mathrm{c}}\right]}_{X 7} k_{t} y_{t}^{n} \\
& +\underbrace{\left[\frac{\alpha}{1-\beta(1-\delta)}\right]\left[\frac{\alpha(1+s)}{1-\alpha}-\frac{\sigma}{\mathrm{c}} \cdot \frac{1-\mathrm{c}}{\delta}(1-\delta)\right]}_{X 8} k_{t} k_{t}^{n}+\underbrace{\left[\frac{\alpha}{1-\beta(1-\delta)}\right]\left[\frac{\sigma}{\mathrm{c}} \cdot \frac{1-\mathrm{c}}{\delta}\right]}_{X 9} k_{t} k_{t+1}^{n} \\
& \underbrace{-\alpha \beta}_{X 10}\left(k_{t+1} y_{t+1}^{n}-k_{t+1} k_{t+1}^{n}\right)+\underbrace{\left[\frac{\alpha \beta}{1-\beta(1-\delta)}\right]\left[\frac{\sigma}{\mathrm{c}}\right]}_{X 11} k_{t+1} y_{t+1}^{n}+\underbrace{\left[\frac{\alpha \beta}{1-\beta(1-\delta)}\right]\left[-\frac{\sigma}{\mathrm{c}}\right]}_{X 12} k_{t+1} y_{t}^{n}
\end{aligned}
$$




$$
\begin{aligned}
& +\underbrace{\left[\frac{\alpha \beta}{1-\beta(1-\delta)}\right]\left[-\frac{\sigma}{\mathrm{c}} \cdot \frac{1-\mathrm{c}}{\delta}\right]}_{X 13} k_{t+1} k_{t+2}^{n}+\underbrace{\left[\frac{\alpha \beta}{1-\beta(1-\delta)}\right]\left[\frac{\sigma}{\mathrm{c}} \cdot \frac{1-\mathrm{c}}{\delta}\right]}_{X 14} k_{t+1} k_{t+1}^{n} \\
& +\underbrace{\left[\frac{\alpha \beta}{1-\beta(1-\delta)}\right]\left[\frac{\sigma}{\mathrm{c}} \cdot \frac{1-\mathrm{c}}{\delta}(1-\delta)\right]}_{X 15} k_{t+1} k_{t+1}^{n}+\underbrace{\left[\frac{\alpha \beta}{1-\beta(1-\delta)}\right]\left[-\frac{\sigma}{\mathrm{c}} \cdot \frac{1-\mathrm{c}}{\delta}(1-\delta)\right]}_{X 16} k_{t+1} k_{t}^{n})] \\
& + \text { t.i.p. } \boldsymbol{+} \mathcal{O}\left(\|\xi\|^{3}\right) .
\end{aligned}
$$

We will simplify (72) as much as possible by trying to combine second-order terms in the model variables with terms that are cross products between the variables and natural-rate expressions (i.e., the variables from the flexible-price model). The goal is to "complete the square" across a number of terms, which will then allow the loss function to be expressed in terms of gap variables (which are more easily interpretable).

We can begin to simplify (72) by noting a number of substitutions. (For the latter substitutions in this list we use the fact - noted in equation 45 - that $\frac{1-c}{\delta}=\frac{\beta \alpha}{1-\beta(1-\delta)}$.)

- $X 1=-C 1-H 2 ; X 2=-C 4-H 3 ;$ and $X 3=-C 5$.

- $X 4+X 7=-\alpha\left[\frac{s+\alpha}{1-\alpha}+\frac{\sigma}{c}\right]=Y 1$.

- $X 5+X 8=\alpha\left[\frac{\alpha(1+s)}{1-\alpha}-\frac{\sigma}{c} \cdot \frac{1-c}{\delta}(1-\delta)\right]=Y 2+Y 3$.

- $X 6=\left[\frac{\beta \alpha(1-\delta)}{1-\beta(1-\delta)}\right]\left[-\frac{\sigma}{\mathrm{c}} \cdot \frac{1-\mathrm{c}}{\delta}\right]=\left[\frac{1-\mathrm{c}}{\delta}(1-\delta)\right]\left[-\frac{\sigma}{\mathrm{c}} \cdot \frac{1-\mathrm{c}}{\delta}\right]=-C 6$.

- The term $X 9$ from a period ahead - that is, $\beta X 9(t+1) k_{t+1} k_{t+2}^{n}$-cancels with the $X 13$ term from the current period-that is, $X 13 k_{t+1} k_{t+1}^{n}$.

- $X 12=\left[\frac{\alpha \beta}{1-\beta(1-\delta)}\right]\left[-\frac{\sigma}{\mathrm{c}}\right]=\left[\frac{1-\mathrm{c}}{\delta}\right]\left[-\frac{\sigma}{\mathrm{c}}\right]=-C 5$.

- $X 14=\left[\frac{\alpha \beta}{1-\beta(1-\delta)}\right]\left[\frac{\sigma}{c} \cdot \frac{1-c}{\delta}\right]=\left[\frac{1-c}{\delta}\right]\left[\frac{\sigma}{c} \cdot \frac{1-c}{\delta}\right]=-C 2 b$.

- $X 16=\left[\frac{\beta \alpha}{1-\beta(1-\delta)}\right]\left[-\frac{\sigma}{c} \cdot \frac{1-c}{\delta}(1-\delta)\right]=\left[\frac{1-c}{\delta}\right]\left[-\frac{\sigma}{c} \cdot \frac{1-c}{\delta}(1-\delta)\right]=-C 6$.

Any new coefficients introduced in this round of substitutions will be labeled $Y$. Now that we have defined all of our $C$ and $H$ coefficients, to conserve on space we will replace the coefficient values with these names. This yields:

$$
\left(\frac{C_{*}^{1-\sigma}}{\mathrm{c}}\right)^{-1} E_{0}\left[\sum_{t=0}^{\infty} \beta^{t}\left(\frac{1}{1-\sigma}\left(C_{t}\right)^{1-\sigma} e^{a \chi_{t}^{c}}-\frac{1}{1+s}\left(H_{t}\right)^{1+s} e^{b \chi_{t}^{h}}\right)\right]
$$




$$
\begin{aligned}
=E_{0}\left[\sum_{t=0}^{\infty} \beta^{t}\right. & \left(\frac{1}{2}[C 1] y_{t}^{2}+\frac{1}{2}([C 2 a]+[C 2 b]) k_{t+1}^{2}+\frac{1}{2}([C 3 a]+[C 3 b]) k_{t}^{2}+[C 4] y_{t} k_{t}+[C 5] y_{t} k_{t+1}\right. \\
& +[C 6] k_{t} k_{t+1}+\frac{1}{2}[H 1] k_{t}^{2}+\frac{1}{2}[H 2] y_{t}^{2}+[H 3] k_{t} y_{t}-\frac{1}{2} \cdot \frac{1}{1-\alpha}\left(\frac{1}{\theta}+\frac{\alpha}{1-\alpha}\right) \operatorname{var}_{z} y_{t}(z) \\
& +[-C 1-H 2] y_{t} y_{t}^{n}+[-C 4-H 3] y_{t} k_{t}^{n}+[-C 5] y_{t} k_{t+1}^{n}+\underbrace{\alpha\left[-\frac{s+\alpha}{1-\alpha}-\frac{\sigma}{\mathrm{c}}\right]}_{Y 1} k_{t} y_{t}^{n} \\
& +\underbrace{\left[\frac{\alpha^{2}(1+s)}{1-\alpha}\right]}_{X 11} k_{t} k_{t}^{n}+\underbrace{\left[-\alpha \frac{\sigma}{\mathrm{C}} \cdot \frac{1-\mathrm{c}}{\delta}(1-\delta)\right]}_{X 10} k_{t} k_{t}^{n}+[-C 6] k_{t} k_{t+1}^{n}+\underbrace{\alpha \beta}_{X 10}\left(k_{t+1} y_{t+1}^{n}-k_{t+1} k_{t+1}^{n}\right) \\
& +\underbrace{\left[\frac{\alpha \beta}{1-\beta(1-\delta)}\right]\left[\frac{\sigma}{\mathrm{c}}\right]}_{X 15} k_{t+1} y_{t+1}^{n}+[-C 5] k_{t+1} y_{t}^{n}+[-C 2 b] k_{t+1} k_{t+1}^{n} \\
& +\underbrace{\left[\frac{\alpha \beta}{1-\beta(1-\delta)}\right]\left[\frac{\sigma}{\mathrm{c}} \cdot \frac{1-\mathrm{c}}{\delta}(1-\delta)\right]}_{X \beta} k_{t+1} k_{t+1}^{n}+[-C 6] k_{t+1} k_{t}^{n})]+ \text { t.i.p. }+\mathcal{O}\left(\|\xi\|^{3}\right) .
\end{aligned}
$$

We can simplify (73) by noting that:

- $Y 1=-\alpha \frac{1+s}{1-\alpha}+\alpha-\alpha \frac{\sigma}{\mathrm{c}}=Z 1+Z 2+Z 3$ (terms in the next equation).

- $Y 2=-H 1$.

Any new coefficients introduced in this round of substitution will be labeled $Z$. We obtain:

$$
\begin{aligned}
\left(\frac{C_{*}^{1-\sigma}}{\mathrm{c}}\right)^{-1} & E_{0}\left[\sum_{t=0}^{\infty} \beta^{t}\left(\frac{1}{1-\sigma}\left(C_{t}\right)^{1-\sigma} e^{a \chi_{t}^{c}}-\frac{1}{1+s}\left(H_{t}\right)^{1+s} e^{b \chi_{t}^{h}}\right)\right] \\
=E_{0}\left[\sum_{t=0}^{\infty} \beta^{t}\right. & \left(\frac{1}{2}[C 1] y_{t}^{2}+\frac{1}{2}([C 2 a]+[C 2 b]) k_{t+1}^{2}+\frac{1}{2}([C 3 a]+[C 3 b]) k_{t}^{2}+[C 4] y_{t} k_{t}+[C 5] y_{t} k_{t+1}\right. \\
& +[C 6] k_{t} k_{t+1}+\frac{1}{2}[H 1] k_{t}^{2}+\frac{1}{2}[H 2] y_{t}^{2}+[H 3] k_{t} y_{t}-\frac{1}{2} \cdot \frac{1}{1-\alpha}\left(\frac{1}{\theta}+\frac{\alpha}{1-\alpha}\right) \operatorname{var}_{z} y_{t}(z) \\
& +[-C 1-H 2] y_{t} y_{t}^{n}+[-C 4-H 3] y_{t} k_{t}^{n}+[-C 5] y_{t} k_{t+1}^{n}+\underbrace{\left[-\alpha \frac{1+s}{1-\alpha}\right]}_{Z 1} k_{t} y_{t}^{n}+\underbrace{[\alpha]}_{Z 2} k_{t} y_{t}^{n} \\
& +\underbrace{\left[-\alpha \frac{\sigma}{\mathrm{c}}\right]}_{Z 3} k_{t} y_{t}^{n}+[-H 1] k_{t} k_{t}^{n}+\underbrace{\left[-\alpha \frac{\sigma}{\mathrm{c}} \cdot \frac{1-\mathrm{c}}{\delta}(1-\delta)\right]}_{Y 3} k_{t} k_{t}^{n}+[-C 6] k_{t} k_{t+1}^{n} \underbrace{-\alpha \beta}_{X 10 a} k_{t+1} y_{t+1}^{n} \\
& \underbrace{-\alpha \beta}_{X 10 b} k_{t+1} k_{t+1}^{n}+\underbrace{\left[\frac{\alpha \beta}{1-\beta(1-\delta)}\right]\left[\frac{\sigma}{\mathrm{c}}\right]}_{X 11} k_{t+1} y_{t+1}^{n}+[-C 5] k_{t+1} y_{t}^{n}+[-C 2 b] k_{t+1} k_{t+1}^{n}
\end{aligned}
$$




$$
+\underbrace{\left[\frac{\alpha \beta}{1-\beta(1-\delta)}\right]\left[\frac{\sigma}{\mathrm{c}} \cdot \frac{1-\mathrm{c}}{\delta}(1-\delta)\right]}_{X 15} k_{t+1} k_{t+1}^{n}+[-C 6] k_{t+1} k_{t}^{n})]+ \text { t.i.p. }+\mathcal{O}\left(\|\xi\|^{3}\right) .
$$

We can simplify (74) by noting that:

- $Z 1=-H 3$.

- The term $Z 2$ from a period ahead - that is $\beta Z 2(t+1) k_{t+1} y_{t+1}^{n}$ - cancels with the $X 10 a$ term from the current period- that is $X 10 a k_{t+1} y_{t+1}^{n}$.

- The term $X 11$ from one period earlier - that is, $\frac{1}{\beta} X 11 k_{t} y_{t}^{n}$ - can be moved one period later and combined with $Z 3$ from the current period - that is, $Z 3 k_{t} y_{t}^{n}$ - to yield $-\frac{\alpha \sigma}{c}\left(1-\frac{1}{1-\beta(1-\delta)}\right) k_{t} y_{t}^{n}=\frac{\alpha \sigma}{c}\left(\frac{\beta(1-\delta)}{1-\beta(1-\delta)}\right) k_{t} y_{t}^{n}=\frac{\sigma}{c}\left(\frac{\alpha \beta(1-\delta)}{1-\beta(1-\delta)}\right) k_{t} y_{t}^{n}=\frac{\sigma}{c} \cdot \frac{1-c}{\delta}(1-$ $\delta) k_{t} y_{t}^{n}=-C 4 k_{t} y_{t}^{n}$. (In the very initial period the term $k_{0} y_{0}^{n}$, which is independent of policy, can be added to the expression so that the within-period loss function looks the same for all periods.)

- The term $X 15$ from one period earlier - that is, $\frac{1}{\beta} X 15 k_{t} k_{t}^{n}$ - can be moved one period later and combined with $Y 3$ from the current period - that is, $Y 3 k_{t} k_{t}^{n}$ - to yield $\left[-\frac{\alpha \sigma}{\mathrm{c}} \cdot \frac{1-\mathrm{c}}{\delta}(1-\delta)\right]\left[1-\frac{1}{1-\beta(1-\delta)}\right] k_{t} k_{t}^{n}=\left[\frac{\sigma}{\mathrm{c}} \cdot \frac{1-\mathrm{c}}{\delta}(1-\delta)\right]\left[\frac{\alpha \beta(1-\delta)}{1-\beta(1-\delta)}\right] k_{t} k_{t}^{n}=\frac{\sigma}{\mathrm{c}}\left(\frac{1-\mathrm{c}}{\delta}\right)^{2}(1-$ $\delta)^{2} k_{t} k_{t}^{n}=-C 3 b k_{t} k_{t}^{n}$. (In the very initial period, the term $k_{0} k_{0}^{n}$, which is independent of policy, can be added to the period so that the within-period loss function looks the same for all periods.)

This implies:

$$
\begin{aligned}
\left(\frac{C_{*}^{1-\sigma}}{c}\right)^{-1} & E_{0}\left[\sum_{t=0}^{\infty} \beta^{t}\left(\frac{1}{1-\sigma}\left(C_{t}\right)^{1-\sigma} e^{a \chi_{t}^{c}}-\frac{1}{1+s}\left(H_{t}\right)^{1+s} e^{b \chi_{t}^{h}}\right)\right] \\
=E_{0}\left[\sum_{t=0}^{\infty} \beta^{t}\right. & \left(\frac{1}{2}[C 1] y_{t}^{2}+\frac{1}{2}([C 2 a]+[C 2 b]) k_{t+1}^{2}+\frac{1}{2}([C 3 a]+[C 3 b]) k_{t}^{2}+[C 4] y_{t} k_{t}+[C 5] y_{t} k_{t+1}\right. \\
& +[C 6] k_{t} k_{t+1}+\frac{1}{2}[H 1] k_{t}^{2}+\frac{1}{2}[H 2] y_{t}^{2}+[H 3] k_{t} y_{t}-\frac{1}{2} \cdot \frac{1}{1-\alpha}\left(\frac{1}{\theta}+\frac{\alpha}{1-\alpha}\right) v_{a} r_{z} y_{t}(z) \\
& +[-C 1-H 2] y_{t} y_{t}^{n}+[-C 4-H 3] y_{t} k_{t}^{n}+[-C 5] y_{t} k_{t+1}^{n}+[-H 3] k_{t} y_{t}^{n}+[-C 4] k_{t} y_{t}^{n} \\
& +[-H 1] k_{t} k_{t}^{n}+[-C 3 b] k_{t} k_{t}^{n}+[-C 6] k_{t} k_{t+1}^{n}-\alpha \beta k_{t+1} k_{t+1}^{n}+[-C 5] k_{t+1} y_{t}^{n} \\
& \left.\left.+[-C 2 b] k_{t+1} k_{t+1}^{n}+[-C 6] k_{t+1} k_{t}^{n}\right)\right]+ \text { t.i.p. }+\mathcal{O}\left(\|\xi\|^{3}\right) .
\end{aligned}
$$


The exercise now is to regroup terms so that we can interpret our loss function in terms of gaps between our sticky- and flexible-price model variables:

$$
\begin{aligned}
\left(\frac{C_{*}^{1-\sigma}}{\mathrm{c}}\right)^{-1} & E_{0}\left[\sum_{t=0}^{\infty} \beta^{t}\left(\frac{1}{1-\sigma}\left(C_{t}\right)^{1-\sigma} e^{a \chi_{t}^{c}}-\frac{1}{1+s}\left(H_{t}\right)^{1+s} e^{b \chi_{t}^{h}}\right)\right] \\
=E_{0}\left[\sum_{t=0}^{\infty} \beta^{t}\right. & \left(\frac{1}{2}[C 1+H 2]\left(y_{t}^{2}-2 y_{t} y_{t}^{n}\right)+\frac{1}{2}[C 2 b]\left(k_{t+1}^{2}-2 k_{t+1} k_{t+1}^{n}\right)+\frac{1}{2}[C 3 b+H 1]\left(k_{t}^{2}-2 k_{t} k_{t}^{n}\right)\right. \\
& +[C 4+H 3]\left(y_{t} k_{t}-y_{t} k_{t}^{n}-k_{t} y_{t}^{n}\right)+[C 5]\left(y_{t} k_{t+1}-y_{t} k_{t+1}^{n}-k_{t+1} y_{t}^{n}\right) \\
& +[C 6]\left(k_{t} k_{t+1}-k_{t} k_{t+1}^{n}-k_{t+1} k_{t}^{n}\right)+\frac{1}{2}[C 2 a] k_{t+1}^{2}+\frac{1}{2}[C 3 a] k_{t}^{2}+\alpha \beta k_{t+1} k_{t+1}^{n} \\
& \left.\left.-\frac{1}{2} \cdot \frac{1}{1-\alpha}\left(\frac{1}{\theta}+\frac{\alpha}{1-\alpha}\right) \operatorname{var}_{z} y_{t}(z)\right)\right]+ \text { t.i.p. }+\mathcal{O}\left(\|\xi\|^{3}\right) .
\end{aligned}
$$

Only the terms $\frac{1}{2}[C 2 a] k_{t+1}^{2}+\frac{1}{2}[C 3 a] k_{t}^{2}+\alpha \beta k_{t+1} k_{t+1}^{n}$ remain ungrouped in equation (76). These terms can be rearranged, however, by noting that the term $C 3 a$ from one period ahead - that is, $\beta C 3 a k_{t+1}^{2}$ - can be moved back a period and combined with the term $C 2 a$ from the current period (that is, $\left.C 2 a k_{t+1}^{2}\right)$ to yield $-\frac{1-c}{\delta}(1-\beta(1-\delta)) k_{t+1}^{2}=-\frac{\alpha \beta}{1-\beta(1-\delta)}(1-$ $\beta(1-\delta)) k_{t+1}^{2}=-\alpha \beta k_{t+1}^{2}$. Thus, by shifting terms from one period to another, we can replace $\frac{1}{2}[C 2 a] k_{t+1}^{2}+\frac{1}{2}[C 3 a] k_{t}^{2}+\alpha \beta k_{t+1} k_{t+1}^{n}$ with $-\frac{1}{2} \alpha \beta\left(k_{t+1}^{2}-2 k_{t+1} k_{t+1}^{n}\right)$. Then equation (76) becomes:

$$
\begin{aligned}
\left(\frac{C_{*}^{1-\sigma}}{\mathrm{c}}\right)^{-1} & E_{0}\left[\sum_{t=0}^{\infty} \beta^{t}\left(\frac{1}{1-\sigma}\left(C_{t}\right)^{1-\sigma} e^{a \chi_{t}^{c}}-\frac{1}{1+s}\left(H_{t}\right)^{1+s} e^{b \chi_{t}^{h}}\right)\right] \\
=E_{0}\left[\sum_{t=0}^{\infty} \beta^{t}\right. & \left(\frac{1}{2}[C 1+H 2]\left(y_{t}^{2}-2 y_{t} y_{t}^{n}\right)+\frac{1}{2}[C 2 b]\left(k_{t+1}^{2}-2 k_{t+1} k_{t+1}^{n}\right)+\frac{1}{2}[C 3 b+H 1]\left(k_{t}^{2}-2 k_{t} k_{t}^{n}\right)\right. \\
& +[C 4+H 3]\left(y_{t} k_{t}-y_{t} k_{t}^{n}-k_{t} y_{t}^{n}\right)+[C 5]\left(y_{t} k_{t+1}-y_{t} k_{t+1}^{n}-k_{t+1} y_{t}^{n}\right) \\
& +[C 6]\left(k_{t} k_{t+1}-k_{t} k_{t+1}^{n}-k_{t+1} k_{t}^{n}\right)-\frac{1}{2} \alpha \beta\left(k_{t+1}^{2}-k_{t+1} k_{t+1}^{n}\right) \\
& \left.\left.-\frac{1}{2} \cdot \frac{1}{1-\alpha}\left(\frac{1}{\theta}+\frac{\alpha}{1-\alpha}\right) \operatorname{var}_{z} y_{t}(z)\right)\right]+ \text { t.i.p. }+\mathcal{O}\left(\|\xi\|^{3}\right) .
\end{aligned}
$$

It is straightforward to complete the relevant quadratics with natural-rate terms that are independent of policy. We also substitute for $C 1, C 2 b, C 3 b, C 4, C 5, C 6, H 1, H 2$, and $H 3$, and replace $(1-\mathrm{c}) / \delta$ using equation (45). Doing so yields:

$$
\begin{aligned}
& \left(\frac{C_{*}^{1-\sigma}}{\mathrm{c}}\right)^{-1} E_{0}\left[\sum_{t=0}^{\infty} \beta^{t}\left(\frac{1}{1-\sigma}\left(C_{t}\right)^{1-\sigma} e^{a \chi_{t}^{c}}-\frac{1}{1+s}\left(H_{t}\right)^{1+s} e^{b \chi_{t}^{h}}\right)\right] \\
= & E_{0}\left[\sum _ { t = 0 } ^ { \infty } \beta ^ { t } \left(-\frac{1}{2}\left[\frac{s+\alpha}{1-\alpha}+\frac{\sigma}{\mathrm{c}}\right]\left(y_{t}-y_{t}^{n}\right)^{2}-\frac{1}{2}\left[\frac{\sigma}{\mathrm{c}}\left(\frac{\alpha \beta}{1-\beta(1-\delta)}\right)^{2}+\alpha \beta\right]\left(k_{t+1}-k_{t+1}^{n}\right)^{2}\right.\right.
\end{aligned}
$$




$$
\begin{aligned}
& -\frac{1}{2}\left[\frac{\sigma}{\mathrm{c}}\left(\frac{\alpha \beta(1-\delta)}{1-\beta(1-\delta)}\right)^{2}+\frac{\alpha^{2}(1+s)}{1-\alpha}\right]\left(k_{t}-k_{t}^{n}\right)^{2}-\frac{1}{2} \cdot \frac{1}{1-\alpha}\left(\frac{1}{\theta}+\frac{\alpha}{1-\alpha}\right) \operatorname{var}_{z} y_{t}(z), \\
& +\left[-\frac{\sigma}{\mathrm{c}} \cdot \frac{\alpha \beta(1-\delta)}{1-\beta(1-\delta)}+\frac{\alpha(1+s)}{1-\alpha}\right]\left(y_{t}-y_{t}^{n}\right)\left(k_{t}-k_{t}^{n}\right) \\
& +\left[\frac{\sigma}{\mathrm{c}} \cdot \frac{\alpha \beta}{1-\beta(1-\delta)}\right]\left(y_{t}-y_{t}^{n}\right)\left(k_{t+1}-k_{t+1}^{n}\right) \\
& \left.\left.+\left[\frac{\sigma}{\mathrm{c}}\left(\frac{\alpha \beta}{1-\beta(1-\delta)}\right)^{2}(1-\delta)\right]\left(k_{t}-k_{t}^{n}\right)\left(k_{t+1}-k_{t+1}^{n}\right)\right)\right]+ \text { t.i.p. }+\mathcal{O}\left(\|\xi\|^{3}\right) .
\end{aligned}
$$

This is equation (58) from section 5.3.2.

\section{A.2 Detailing the Steps between Equations (56) and (57)}

I label all terms in (56) in the same manner that (53) was labeled.

$$
\begin{aligned}
& \left(\frac{C_{*}^{1-\sigma}}{\mathrm{c}}\right)^{-1} E_{0}\left[\sum_{t=0}^{\infty} \beta^{t}\left(\frac{1}{1-\sigma}\left(C_{t}\right)^{1-\sigma} e^{a \chi_{t}^{c}}-\frac{1}{1+s}\left(H_{t}\right)^{1+s} e^{b \chi_{t}^{h}}\right)\right]
\end{aligned}
$$

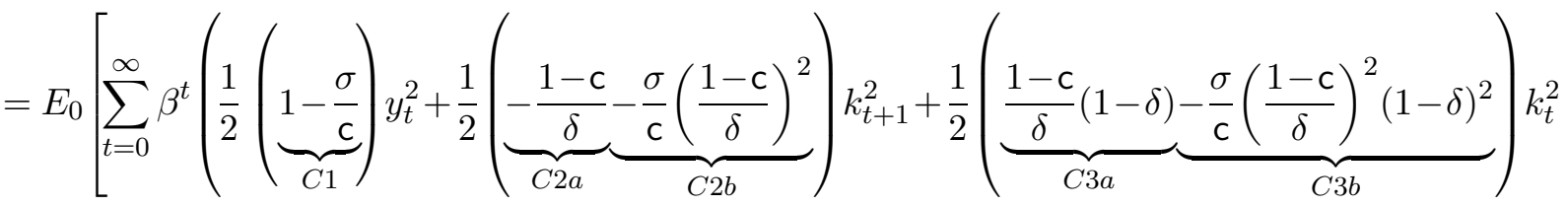

$$
\begin{aligned}
& \underbrace{-\frac{\sigma}{\mathrm{c}} \cdot \frac{1-\mathrm{c}}{\delta}(1-\delta)}_{C 4} y_{t} k_{t}+\underbrace{\frac{\sigma}{\mathrm{c}} \cdot \frac{1-\mathrm{c}}{\delta}}_{C 5} y_{t} k_{t+1}+\underbrace{\frac{\sigma}{\mathrm{c}\left(\frac{1-\mathrm{c}}{\delta}\right)^{2}(1-\delta)}}_{C 6} k_{t} k_{t+1}+\frac{1}{2} \cdot \underbrace{\frac{-\alpha}{1-\alpha} \alpha(1+s)}_{H 1} k_{t}^{2} \\
& +\frac{1}{2} \cdot \underbrace{\frac{-(1+s)}{1-\alpha}}_{H 2} y_{t}^{2}+\underbrace{\frac{\alpha(1+s)}{1-\alpha}}_{H 3} k_{t} y_{t}-\frac{1}{2} \cdot \frac{1}{1-\alpha}\left(\frac{1}{\theta}+\frac{\alpha}{1-\alpha}\right) \operatorname{var}_{z} y_{t}(z) \\
& +\underbrace{\left[\frac{s+\alpha}{1-\alpha}+\frac{\sigma}{\mathrm{c}}\right]}_{X 1} y_{t} y_{t}^{n}+\underbrace{\left[-\frac{\alpha(1+s)}{1-\alpha}+\frac{\sigma}{\mathrm{c}} \cdot \frac{1-\mathrm{c}}{\delta}(1-\delta)\right]}_{X 2} y_{t} k_{t}+\underbrace{\left[-\frac{\sigma}{\mathrm{c}} \cdot \frac{1-\mathrm{c}}{\delta}\right]}_{X 3} y_{t} k_{t+1}^{n} \\
& +\underbrace{\left[\frac{\beta \alpha(1-\delta)}{1-\beta(1-\delta)}\right]\left[\frac{s+\alpha}{1-\alpha}+\frac{\sigma}{\mathrm{c}}\right]}_{X 4} k_{t} y_{t}^{n}+\underbrace{\left[\frac{\beta \alpha(1-\delta)}{1-\beta(1-\delta)}\right]\left[-\frac{\alpha(1+s)}{1-\alpha}+\frac{\sigma}{\mathrm{c}} \cdot \frac{1-\mathrm{c}}{\delta}(1-\delta)\right]}_{X 5} k_{t}^{2} \\
& +\underbrace{\left[\frac{\beta \alpha(1-\delta)}{1-\beta(1-\delta)}\right]\left[-\frac{\sigma}{\mathrm{c}} \cdot \frac{1-\mathrm{c}}{\delta}\right]}_{X 6} k_{t} k_{t+1}^{n}+\underbrace{\left[\frac{\alpha}{1-\beta(1-\delta)}\right]\left[-\frac{s+\alpha}{1-\alpha}-\frac{\sigma}{\mathrm{c}}\right]}_{X 7} k_{t} y_{t}^{n} \\
& +\underbrace{\left[\frac{\alpha}{1-\beta(1-\delta)}\right]\left[\frac{\alpha(1+s)}{1-\alpha}-\frac{\sigma}{\mathrm{c}} \cdot \frac{1-\mathrm{c}}{\delta}(1-\delta)\right]}_{X 8} k_{t}^{2}+\underbrace{\left[\frac{\alpha}{1-\beta(1-\delta)}\right]\left[\frac{\sigma}{\mathrm{c}} \cdot \frac{1-\mathrm{c}}{\delta}\right]}_{X 9} k_{t} k_{t+1}^{n}
\end{aligned}
$$




$$
\begin{aligned}
& \underbrace{-\alpha \beta}_{X 10}\left(k_{t+1} y_{t+1}^{n}-k_{t+1} k_{t+1}^{n}\right)+\underbrace{\left[\frac{\alpha \beta}{1-\beta(1-\delta)}\right]\left[\frac{\sigma}{\mathrm{c}}\right]}_{X 11} k_{t+1} y_{t+1}^{n}+\underbrace{\left[\frac{\alpha \beta}{1-\beta(1-\delta)}\right]\left[-\frac{\sigma}{\mathrm{c}}\right]}_{X 13} k_{t+1} y_{t}^{n} \\
& +\underbrace{\left[\frac{\alpha \beta}{1-\beta(1-\delta)}\right]\left[-\frac{\sigma}{\mathrm{c}} \cdot \frac{1-\mathrm{c}}{\delta}\right]}_{X 14} k_{t+1} k_{t+2}^{n}+\underbrace{\left[\frac{\alpha \beta}{1-\beta(1-\delta)}\right]\left[\frac{\sigma}{\left.\mathrm{c} \cdot \frac{1-\mathrm{c}}{\delta}\right]} k_{t+1}\right.}_{X 15} k_{t+1}^{n} \\
& +\underbrace{\left[\frac{\alpha \beta}{1-\beta(1-\delta)}\right]\left[\frac{\sigma}{\mathrm{c}} \cdot \frac{1-\mathrm{c}}{\delta}(1-\delta)\right]}_{X 16} k_{t+1}^{2}+\underbrace{\left[\frac{\alpha \beta}{1-\beta(1-\delta)}\right]\left[-\frac{\sigma}{\mathrm{c}} \cdot \frac{1-\mathrm{c}}{\delta}(1-\delta)\right]}_{X 15} k_{t+1} k_{t})] \\
& + \text { t.i.p. }+\mathcal{O}\left(\|\xi\|^{3}\right)
\end{aligned}
$$

The sequence of simplifying steps listed after equations (72), (73), and (74) can be used to simplify equation (78). Together these imply:

$$
\begin{aligned}
\left(\frac{C_{*}^{1-\sigma}}{c}\right)^{-1} & E_{0}\left[\sum_{t=0}^{\infty} \beta^{t}\left(\frac{1}{1-\sigma}\left(C_{t}\right)^{1-\sigma} e^{a \chi_{t}^{c}}-\frac{1}{1+s}\left(H_{t}\right)^{1+s} e^{b \chi_{t}^{h}}\right)\right] \\
=E_{0}\left[\sum_{t=0}^{\infty} \beta^{t}\right. & \left(\frac{1}{2}[C 1] y_{t}^{2}+\frac{1}{2}([C 2 a]+[C 2 b]) k_{t+1}^{2}+\frac{1}{2}([C 3 a]+[C 3 b]) k_{t}^{2}+[C 4] y_{t} k_{t}+[C 5] y_{t} k_{t+1}\right. \\
& +[C 6] k_{t} k_{t+1}+\frac{1}{2}[H 1] k_{t}^{2}+\frac{1}{2}[H 2] y_{t}^{2}+[H 3] k_{t} y_{t}-\frac{1}{2} \cdot \frac{1}{1-\alpha}\left(\frac{1}{\theta}+\frac{\alpha}{1-\alpha}\right) v_{a r} y_{t}(z) \\
& +[-C 1-H 2] y_{t} y_{t}^{n}+[-C 4-H 3] y_{t} k_{t}+[-C 5] y_{t} k_{t+1}^{n}+[-H 3] k_{t} y_{t}^{n}+[-C 4] k_{t} y_{t}^{n} \\
& +[-H 1] k_{t}^{2}+[-C 6] k_{t} k_{t+1}^{n}-\alpha \beta k_{t+1} k_{t+1}^{n}+[-C 5] k_{t+1} y_{t}^{n}+[-C 2 b] k_{t+1} k_{t+1}^{n} \\
& \left.\left.+[-C 6] k_{t+1} k_{t}+[-C 3 b] k_{t}^{2}\right)\right]+ \text { t.i.p. }+\mathcal{O}\left(\|\xi\|^{3}\right) .
\end{aligned}
$$

As before, we regroup terms so that we can interpret our loss function in terms of gaps between the sticky-price and flexible-price model variables. In doing so, we also use the fact (used earlier) that the $C 3 a$ term from a period earlier - that is, $\beta C 3 a k_{t+1}^{2}$-can be combined with the $C 2 a$ term from the current period - that is $C 2 a k_{t+1}^{2}$ - to yield $-\alpha \beta k_{t+1}^{2}$ :

$$
\begin{aligned}
\left(\frac{C_{*}^{1-\sigma}}{\mathrm{c}}\right)^{-1} & E_{0}\left[\sum_{t=0}^{\infty} \beta^{t}\left(\frac{1}{1-\sigma}\left(C_{t}\right)^{1-\sigma} e^{a \chi_{t}^{c}}-\frac{1}{1+s}\left(H_{t}\right)^{1+s} e^{b \chi_{t}^{h}}\right)\right] \\
=E_{0}\left[\sum_{t=0}^{\infty} \beta^{t}\right. & \left(\frac{1}{2}[C 1+H 2]\left(y_{t}^{2}-2 y_{t} y_{t}^{n}\right)+\frac{1}{2}[C 2 b-\alpha \beta]\left(k_{t+1}^{2}-2 k_{t+1} k_{t+1}^{n}\right)\right. \\
& -\frac{1}{2}[C 3 b+H 1] k_{t}^{2}-[C 4+H 3] k_{t} y_{t}^{n}+[C 5]\left(y_{t} k_{t+1}-y_{t} k_{t+1}^{n}-k_{t+1} y_{t}^{n}\right) \\
& \left.\left.+[C 6] k_{t} k_{t+1}^{n}-\frac{1}{2} \cdot \frac{1}{1-\alpha}\left(\frac{1}{\theta}+\frac{\alpha}{1-\alpha}\right) \operatorname{var}_{z} y_{t}(z)\right)\right]+ \text { t.i.p. }+\mathcal{O}\left(\|\xi\|^{3}\right) .
\end{aligned}
$$


We can then complete the relevant quadratics with natural-rate terms that are independent of policy, substitute for $C 1, C 2 b, C 3 b, C 4, C 5, C 6, H 1, H 2$, and $H 3$, and replace $(1-\mathrm{c}) / \delta$ using equation (45). Doing so yields:

$$
\begin{aligned}
\left(\frac{C_{*}^{1-\sigma}}{\mathrm{c}}\right)^{-1} & E_{0}\left[\sum_{t=0}^{\infty} \beta^{t}\left(\frac{1}{1-\sigma}\left(C_{t}\right)^{1-\sigma} e^{a \chi_{t}^{c}}-\frac{1}{1+s}\left(H_{t}\right)^{1+s} e^{b \chi_{t}^{h}}\right)\right] \\
=E_{0}\left[\sum_{t=0}^{\infty} \beta^{t}(\right. & -\frac{1}{2}\left[\frac{s+\alpha}{1-\alpha}+\frac{\sigma}{\mathrm{c}}\right]\left(y_{t}-y_{t}^{n}\right)^{2}-\frac{1}{2}\left[\frac{\sigma}{\mathrm{c}}\left(\frac{\alpha \beta}{1-\beta(1-\delta)}\right)^{2}+\alpha \beta\right]\left(k_{t+1}-k_{t+1}^{n}\right)^{2} \\
& +\frac{1}{2}\left[\frac{\sigma}{\mathrm{c}}\left(\frac{\alpha \beta(1-\delta)}{1-\beta(1-\delta)}\right)^{2}+\frac{\alpha^{2}(1+s)}{1-\alpha}\right] k_{t}^{2}-\frac{1}{2} \cdot \frac{1}{1-\alpha}\left(\frac{1}{\theta}+\frac{\alpha}{1-\alpha}\right) \operatorname{var}_{z} y_{t}(z) \\
+ & {\left[\frac{\sigma}{\mathrm{c}} \cdot \frac{\alpha \beta(1-\delta)}{1-\beta(1-\delta)}-\frac{\alpha(1+s)}{1-\alpha}\right] k_{t} y_{t}^{n}-\left[\frac{\sigma}{\mathrm{c}}\left(\frac{\alpha \beta}{1-\beta(1-\delta)}\right)^{2}(1-\delta)\right] k_{t} k_{t+1}^{n} } \\
& \left.\left.+\left[\frac{\sigma}{\mathrm{c}} \cdot \frac{\alpha \beta}{1-\beta(1-\delta)}\right]\left(y_{t}-y_{t}^{n}\right)\left(k_{t+1}-k_{t+1}^{n}\right)\right)\right]+ \text { t.i.p. }+\mathcal{O}\left(\|\xi\|^{3}\right) .
\end{aligned}
$$

In terms of the coefficients, the above equation - which is equation (57) in the text-looks a lot like (54); however, not all squares in the above expression have been completed and not everything is in gap form. Thus, we re-write equation (57) as:

$$
\begin{aligned}
\left(\frac{C_{*}^{1-\sigma}}{\mathrm{c}}\right)^{-1} & E_{0}\left[\sum_{t=0}^{\infty} \beta^{t}\left(\frac{1}{1-\sigma}\left(C_{t}\right)^{1-\sigma} e^{a \chi_{t}^{c}}-\frac{1}{1+s}\left(H_{t}\right)^{1+s} e^{b \chi_{t}^{h}}\right)\right] \\
=E_{0}\left[\sum _ { t = 0 } ^ { \infty } \beta ^ { t } \left(-\frac{1}{2}\left[\frac{s+\alpha}{1-\alpha}+\frac{\sigma}{\mathrm{c}}\right]\left(y_{t}-y_{t}^{n}\right)^{2}-\frac{1}{2}\left[\frac{\sigma}{\mathrm{c}}\left(\frac{\alpha \beta}{1-\beta(1-\delta)}\right)^{2}+\alpha \beta\right]\left(k_{t+1}-k_{t+1}^{n}\right)^{2}\right.\right. & -\frac{1}{2}\left[\frac{\sigma}{\mathrm{c}}\left(\frac{\alpha \beta(1-\delta)}{1-\beta(1-\delta)}\right)^{2}+\frac{\alpha^{2}(1+s)}{1-\alpha}\right] k_{t}^{2}+\underbrace{\left[\frac{\sigma}{\mathrm{c}}\left(\frac{\alpha \beta(1-\delta)}{1-\beta(1-\delta)}\right)^{2}\right]}_{A 1} k_{t}^{2}+\underbrace{\left[\frac{\alpha^{2}(1+s)}{1-\alpha}\right]}_{A 2} k_{t}^{2} \\
& -\frac{1}{2} \cdot \frac{1}{1-\alpha}\left(\frac{1}{\theta}+\frac{\alpha}{1-\alpha}\right) \operatorname{var}_{z} y_{t}(z)+\underbrace{\left[\frac{\sigma}{\mathrm{c}} \cdot \frac{\alpha \beta(1-\delta)}{1-\beta(1-\delta)}\right]}_{A 3} k_{t} y_{t}^{n}+\underbrace{\left[-\frac{\alpha(1+s)}{1-\alpha}\right]}_{A 4} k_{t} y_{t}^{n} \\
& +\left[\frac{\sigma}{\mathrm{c}} \cdot \frac{\alpha \beta}{1-\beta(1-\delta)}\right]\left(y_{t}-y_{t}^{n}\right)\left(k_{t+1}-k_{t+1}^{n}\right)+\underbrace{\left[-\frac{\sigma}{\mathrm{c}}\left(\frac{\alpha \beta}{1-\beta(1-\delta)}\right)^{2}(1-\delta)\right]}_{A 5} k_{t} k_{t+1}^{n}] \\
+ & \text { t.i.p. }+\mathcal{O}\left(\|\xi\|^{3}\right) .
\end{aligned}
$$

Noting that:

$$
A 3 k_{t} y_{t}^{n}+A 5 k_{t} k_{t+1}^{n}+A 1 k_{t}^{2}=\left[\sigma \frac{\alpha \beta(1-\delta)}{1-\beta(1-\delta)}\right] k_{t} c_{t}^{n} \text { and } A 4 k_{t} y_{t}^{n}+A 2 k_{t}^{2}=[-\alpha(1+s)] k_{t} h_{t}^{n},
$$


and that $\left(k_{t+1}-k_{t+1}^{n}\right)=\delta\left(i_{t}-i_{t}^{n}\right)$, we can write:

$$
\begin{aligned}
&\left(\frac{C_{*}^{1-\sigma}}{\mathrm{c}}\right)^{-1} E_{0}\left[\sum_{t=0}^{\infty} \beta^{t}\left(\frac{1}{1-\sigma}\left(C_{t}\right)^{1-\sigma} e^{a \chi_{t}^{c}}-\frac{1}{1+s}\left(H_{t}\right)^{1+s} e^{b \chi_{t}^{h}}\right)\right] \\
&=E_{0}\left[\sum _ { t = 0 } ^ { \infty } \beta ^ { t } \left(-\frac{1}{2}\left[\frac{s+\alpha}{1-\alpha}+\frac{\sigma}{\mathrm{c}}\right]\left(y_{t}-y_{t}^{n}\right)^{2}-\frac{1}{2}\left[\frac{\sigma}{\mathrm{c}}\left(\frac{\alpha \beta \delta}{1-\beta(1-\delta)}\right)^{2}+\alpha \beta \delta^{2}\right]\left(i_{t}-i_{t}^{n}\right)^{2}\right.\right. \\
&-\frac{1}{2}\left[\frac{\sigma}{\mathrm{c}}\left(\frac{\alpha \beta(1-\delta)}{1-\beta(1-\delta)}\right)^{2}+\frac{\alpha^{2}(1+s)}{1-\alpha}\right] k_{t}^{2}-\frac{1}{2} \cdot \frac{1}{1-\alpha}\left(\frac{1}{\theta}+\frac{\alpha}{1-\alpha}\right) \operatorname{var}_{z} y_{t}(z) \\
&\left.\left.+\left[\frac{\sigma}{\mathrm{c}} \cdot \frac{\alpha \beta \delta}{1-\beta(1-\delta)}\right]\left(y_{t}-y_{t}^{n}\right)\left(i_{t}-i_{t}^{n}\right)+\left[\sigma \frac{\alpha \beta(1-\delta)}{1-\beta(1-\delta)}\right] k_{t} c_{t}^{n}-[\alpha(1+s)] k_{t} h_{t}^{n}\right)\right] \\
&+ \text { t.i.p. }+\mathcal{O}\left(\|\xi\|^{3}\right) .
\end{aligned}
$$

This is equation (58) in the text. 